\title{
Galois modules arising from Faltings's strict modules
}

\author{
V. Abrashkin
}

\begin{abstract}
Suppose that $O=\mathbb{F}_{q}[\pi]$ is a polynomial ring and $R$ is a commutative unitary $O$-algebra. The category of finite flat group schemes over $R$ with a strict action of $O$ was recently introduced by Faltings and appears as an equal characteristic analogue of the classical category of finite flat group schemes in the equal characteristic case. In this paper we obtain a classification of these modules and apply it to prove analogues of properties that were known earlier for classical group schemes.
\end{abstract}

\section{Introduction}

Throughout all of this paper $p$ is a fixed prime number. Suppose that $R$ is a commutative unitary ring and $\mathrm{Gr}_{R}$ is the category of finite flat commutative group schemes over $R$. By definition its objects are $G=\operatorname{Spec} A(G)$, where $A(G)$ is a commutative flat $R$-algebra, which is a locally free $R$-module of finite rank, with the comultiplication $\Delta: A(G) \longrightarrow A(G) \otimes A(G)$, the counit $e: A(G) \longrightarrow R$ and the coinversion $i: A(G) \longrightarrow A(G)$ satisfying well-known axioms. Denote by $\mathrm{Gr}_{R}^{\prime}$ the full subcategory of $\operatorname{Gr}_{R}$ consisting of $p$-group schemes $G$, i.e. such that $G$ is killed by some power of $p \operatorname{id}_{G}$.

If $R=k$ is a perfect field of characteristic $p$, then the objects $G$ of $\mathrm{Gr}_{k}^{\prime}$ can be described in terms of Dieudonne theory, i.e. in terms of finitely generated $W(k)$-modules $M(G)$ with a $\sigma$-linear operator $F$ and a $\sigma^{-1}$-linear operator $V$ such that $F V=V F=p \operatorname{id}_{M(G)} \cdot(W(k)$ is the ring of Witt vectors with coefficients in $k$ and $\sigma$ is its Frobenius automorphism induced by the $p$ th power map in $k$.)

Suppose that $R$ is the valuation ring of a complete discrete valuation field $K$ of characteristic 0 with a perfect residue field $k$ of characteristic $p$ and $e=e\left(K / \mathbb{Q}_{p}\right)$ is the absolute ramification index of $K$. Then the classification of objects of the category $\mathrm{Gr}_{R}^{\prime}$ was made in [Fon75] under the restriction $e=1$ in terms of finite Honda systems (this classification was not complete for $p=2$, for an improved version cf. [Abr87a]). Further progress was made in [Abr90] for $e \leqslant p-1$ (group schemes killed by $p$ ), in [Con99] for $e<p-1$ and, finally, in [Bre00] for an arbitrary $e$. Most interesting arithmetic applications of finite group schemes $G \in \mathrm{Gr}_{R}^{\prime}$ are related to the properties of $\Gamma_{K}=\operatorname{Gal}(\bar{K} / K)$ modules $H=G(\bar{K})$ of their geometric points. We mention the following three results.

(a) Serre's conjecture (proved in [Ray74]). This result describes the action of the inertia subgroup $I_{K} \subset \Gamma_{K}$ on the semi-simple envelope of $H$. It is given by characters $\chi: I_{K} \longrightarrow \bar{k}^{*}$ such that for some $N \in \mathbb{N}, \chi=\chi_{N}^{a}$, where $\chi_{N}(\tau)=\tau\left(\eta_{N}\right) / \eta_{N}, \eta_{N}^{p^{N}-1}=\eta$ is a uniformiser of $K$, and $a=a_{0}+a_{1} p+\cdots+a_{N-1} p^{N-1}$ with $p$-digits $a_{0}, a_{1}, \ldots, a_{N-1} \in[0, e]$.

(b) Ramification estimates. If $p^{M} \mathrm{id}_{G}=0$, then the ramification subgroups $\Gamma_{K}^{(v)}$ of $\Gamma_{K}$ act trivially on $H$ if $v>e(M-1+1 /(p-1))$, cf. [Fon85].

Received 21 December 2004, accepted in final form 21 October 2005.

2000 Mathematics Subject Classification 14L15, 11G09, 11S15.

Keywords: group schemes, Galois modules.

This paper was written as a part of project supported by EPSRC, GR/S72252/01.

This journal is (c) Foundation Compositio Mathematica 2006. 


\section{ABRASHKIN}

(c) Complete description of the $\Gamma_{K}$-module $H$ (under some additional assumptions). If $e=1, p>3$ and the $\mathbb{F}_{p}\left[\Gamma_{K}\right]$-module $H$ satisfies the above Serre's Conjecture and ramification estimates (i.e. the ramification subgroups $\Gamma_{K}^{(v)}$ act trivially on $H$ if $v>1 /(p-1)$ ), then there is an $G \in \mathrm{Gr}_{R}^{\prime}$ such that $H=G\left(K_{\mathrm{sep}}\right)$, cf. [Abr90].

Suppose now that $R$ is an $\mathbb{F}_{p}$-algebra. In this case the category $\operatorname{Gr}_{R}^{\prime}$ is not interesting because it is 'too big'. For example, if $R$ is an integral domain with the fraction field $K$ then any finite continuous $\mathbb{Z}_{p}\left[\Gamma_{K}\right]$-module appears in the form $G\left(K_{\text {sep }}\right)$ for a suitable $G \in \mathrm{Gr}_{R}^{\prime}$. Even if we assume that $R$ is an $O$-algebra, where $O$ is a complete discrete valuation ring (which plays a role of $\mathbb{Z}_{p}$ in the equal characteristic case), then the category $\operatorname{Gr}^{\prime}(O)_{R}$ of all finite flat commutative group schemes with $O$-action is too big for studying special properties of finite subquotients of formal Drinfeld $O$-modules over $R$. This unpleasant situation became completely different when Faltings introduced a new concept of finite flat group schemes with strict $O$-action, cf. [Fal02]. The main idea of Faltings's definition can be explained as follows.

Suppose that $G=\operatorname{Spec} A(G)$ is a finite flat $O$-module over $R$. Present $A(G)$ as a complete intersection, i.e. as a quotient $R\left[X_{1}, \ldots, X_{n}\right] / I$ of a ring of polynomials by an ideal $I$ generated by elements of a regular sequence of length $n$, and define the deformation $A(G)^{b}$ of $A(G)$ as $R\left[X_{1}, \ldots, X_{n}\right] /\left(I \cdot I_{0}\right)$, where $I_{0}=\left(X_{1}, \ldots, X_{n}\right)$. Faltings requires that the $O$-module structure on $G$, which is given by the endomorphisms $[o]: A(G) \longrightarrow A(G), o \in O$, should have an extension to an $O$-module structure of the deformation $\left(A(G), A(G)^{b}\right)$ of the algebra $A(G)$ and this extension must satisfy the condition of strictness. This means that if $o \in O$ and $[o]^{b}: A(G)^{b} \longrightarrow A(G)^{b}$ is an extension of $[o]$, then $[o]^{b}$ must induce the 'scalar' multiplication by $o$ on $I_{0} / I_{0}^{2}$ and $I /\left(I \cdot I_{0}\right)$. This definition gives (in our notation) the category $\operatorname{DGr}(O)_{R}$ of finite strict $O$-modules $\left(G, G^{b}\right)$, where $G^{b}=\operatorname{Spec} A(G)^{b}$, and its objects have many interesting properties discussed in [Fal02]. Note that the condition of strictness can be viewed as the absence of the first obstacle in the problem of embedding given finite flat local $O$-module scheme into a Drinfeld (= formal) $O$-module.

This paper deals with the classification of finite flat commutative group schemes over $R$ with strict $O$-action, where $O=\mathbb{F}_{q}[\pi]$ with fixed variable $\pi$. Note that this approach can be easily 'sheafi-

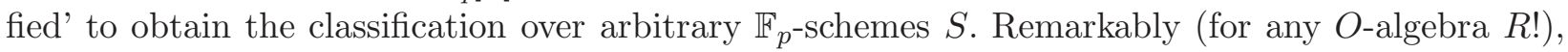
this classification is an almost complete analogue of the classical Dieudonne theory of conventional group schemes over a perfect field of characteristic $p$. Main applications are obtained for the full subcategory $\operatorname{DGr}^{\prime}(O)_{R}$ of $\pi$-torsion group subschemes. In particular, we prove an analogue of Raynaud's theorem, i.e. that locally on $R$ any $\left(G, G^{b}\right) \in \operatorname{DGr}^{\prime}(O)_{R}$ can be embedded into a $\pi$-divisible group consisting of objects of the category $\operatorname{DGr}(O)_{R}$. If $G$ is a local scheme then this gives locally an embedding of $G$ into a formal Drinfeld $O$-module over $R$. In other words, the condition of strictness, when treated as vanishing of the first obstacle for embedding of a local $G$ into a formal $O$-module, appears as a necessary and sufficient condition for the existence of such an embedding. We also study number-theoretic properties of Galois modules $G\left(K_{\mathrm{sep}}\right)$ if $R$ is the valuation ring in a complete discrete valuation field $K$ and $\left(G, G^{b}\right) \in \operatorname{DGr}(O)_{R}$. These properties are precise analogues of the above mentioned properties (a)-(c) of conventional group schemes over complete discrete valuation rings of mixed characteristic.

The paper is structured as follows. In $\S 1$ we recall the concept of a strict $O$-module following the basic idea of Faltings's original definition. In $\S 2$ we describe the category of strict $\mathbb{F}_{q}$-modules over $R$ and apply this in $\S 3$ to classify the objects of the category $\operatorname{DGr}(O)_{R}$ if $O=\mathbb{F}_{q}[\pi]$. This classification is based on the study of primitive elements (i.e. the elements $a \in A(G)$ such that $\Delta a=a \otimes 1+1 \otimes a$, where $\Delta$ is the comultiplication on $G$ ) of the $R$-algebra $A(G)$, where $\left(G, G^{b}\right) \in \operatorname{DGr}(O)_{R}$. In $\S 4$ we apply this classification to prove that any object of $\operatorname{DGr}^{\prime}(O)_{R}$ can be embedded into a $\pi$-divisible group over $R$. This section also contains a comparison of our antiequivalence with the parallel results in the theory of conventional finite flat group schemes and $p$-adic 


\section{FALTings's STRICT MODULES}

representations in the mixed characteristic case from the papers [Abr87a, Abr90, Bre00, Fon90]. In $\S 5$ we establish analogues of the above properties (a), (b) and (c) in the category $\operatorname{DGr}^{\prime}(O)_{R}$, where $R$ is the valuation ring of a complete discrete valuation field $K$ containing the ring $O$.

The author expresses a very deep gratitude to the referee. The original version of this paper dealt only with group schemes over a complete discrete valuation rings of characteristic $p$ with etale generic fibre. The referee proposed to generalize the approach by the use of the classical description of finite flat group schemes having zero Verschiebung from SGA3, cf. [Gab70]. This allowed the author to develop methods from the thesis [Gib04] and to obtain the classification of strict modules over arbitrary ring $R$ of characteristic $p$. The referee report also contains other interesting observations (the author is very sorry to not be able to mention all of them). In particular, in $\S 4.2$ we follow the referee's ideas to prove the following statement conjectured in [Fal02]: if $O=\mathbb{F}_{q}[\pi],\left(G, G^{b}\right) \in$ $\operatorname{DGr}^{\prime}(O)_{R}$ and $\operatorname{rk}_{R} A(G)=q^{h}$, then $G$ is killed by $[\pi]^{h}$.

\section{Notation and conventions}

Everywhere in the paper $p$ is a fixed prime number, $O$ is a unitary commutative $\mathbb{F}_{p}$-algebra (in most cases $O$ is the polynomial ring $\left.\mathbb{F}_{q}[\pi]\right)$ and $R$ is a commutative unitary $O$-algebra. If $f: A \longrightarrow B$ and $g: B \longrightarrow C$ are maps of sets, then we denote their decomposition as $f \circ g$, i.e. for any $a \in A$, $(f \circ g)(a)=g(f(a))$.

\section{Definition and simplest properties}

An $R$-algebra $A$ will be called finite if it is a locally free $R$-module of finite rank.

\subsection{Deformations of augmented $\boldsymbol{R}$-algebras}

For an augmented $O$-algebra $A$, we agree to use the following notation: $\varepsilon_{A}: A \longrightarrow O$, the morphism of augmentation, and $\operatorname{Ker} \varepsilon_{A}=I_{A}$, the augmentation ideal. If $R[\bar{X}]=R\left[X_{1}, \ldots, X_{n}\right], n \geqslant 0$, is a polynomial ring we always assume that its augmentation ideal $I_{R[\bar{X}]}=\left(X_{1}, \ldots, X_{n}\right)$.

The objects of the category $\operatorname{DAug}_{R}$ are the triples $\mathcal{A}=\left(A, A^{b}, i_{\mathcal{A}}\right)$, where $A$ is a finite augmented $R$-algebra, $A^{b}$ is an augmented $R$-algebra and $i_{\mathcal{A}}: A^{b} \longrightarrow A$ is an epimorphic map of augmented $R$-algebras such that there is a polynomial ring $R[\bar{X}]=R\left[X_{1}, \ldots, X_{n}\right], n \geqslant 0$, and an epimorphism of augmented $R$-algebras $j: R[\bar{X}] \longrightarrow A^{b}$ satisfying the following properties:

- the ideal $I:=\operatorname{Ker}\left(j \circ i_{\mathcal{A}}\right)$ is generated by elements of a regular sequence of length $n$ in $R[\bar{X}]$;

- $\operatorname{Ker} j=I \cdot I_{R[\bar{X}]}$.

A morphism $\bar{f}=\left(f, f^{b}\right): \mathcal{A} \longrightarrow \mathcal{B}=\left(B, B^{b}, i_{\mathcal{B}}\right)$ in $\operatorname{DAug}_{R}$ is given by morphisms of augmented $R$-algebras $f: A \rightarrow B$ and $f^{b}: A^{b} \longrightarrow B^{b}$ such that $i_{\mathcal{A}} \circ f=f^{b} \circ i_{\mathcal{B}}$.

Note that if $\left(A, A^{b}, i_{\mathcal{A}}\right) \in \operatorname{DAug}_{R}$, then $A^{\text {b }}$ is a finite $R$-algebra.

Below, we use the simpler notation $\left(A, A^{b}\right)$ instead of $\left(A, A^{b}, i_{\mathcal{A}}\right)$ if it does not lead to a misunderstanding.

In the category $\operatorname{DAug}_{R}, \mathcal{R}=\left(R, R, \mathrm{id}_{R}\right)$ is an initial object and any $\mathcal{A}=\left(A, A^{b}, i_{\mathcal{A}}\right)$ has a natural augmentation to $\mathcal{R}, \varepsilon_{\mathcal{A}}=\left(\varepsilon_{A}, \varepsilon_{A^{b}}\right): \mathcal{A} \longrightarrow \mathcal{R}$, where $\operatorname{Ker} \varepsilon_{A^{b}}=\operatorname{Ann}\left(\operatorname{Ker} i_{\mathcal{A}}\right):=I_{A^{b}}$.

Introduce the $R$-modules $t_{\mathcal{A}}^{*}=I_{R[\bar{X}]} / I_{R[\bar{X}]}^{2}$ and $N_{\mathcal{A}}=I /\left(I \cdot I_{R[\bar{X}]}\right)$. They do not depend on the choice of the above covering $j: R[\bar{X}] \longrightarrow A^{b}$. Indeed, the first coincides with $I_{A^{b}} / I_{A^{b}}^{2}$ and the second with $\operatorname{Ker} i_{\mathcal{A}}$. Note also that both $R$-modules are free. (This is obvious for the first module and follows from the fact that $A$ is a complete intersection for the second.)

If $\mathcal{A}=\left(A, A^{b}\right)$ and $\mathcal{B}=\left(B, B^{b}\right)$ are objects in $\operatorname{DAug}_{R}$ and $f: A \longrightarrow B$ is a morphism of augmented $R$-algebras, then the set of all $f^{b} \operatorname{such}$ that $\left(f, f^{b}\right) \in \operatorname{Hom}_{\mathrm{DAug}}(\mathcal{A}, \mathcal{B})$ is not empty and has a natural structure of a principal homogeneous space over the group $\operatorname{Hom}_{R \text {-mod }}\left(t_{\mathcal{A}}^{*}, N_{\mathcal{B}}\right)$. 


\section{Abrashinin}

Let DAug* be a quotient category for $\operatorname{DAug}_{R}$ : it has the same objects but its morphisms are equivalence classes of morphisms from $\operatorname{Hom}_{\mathrm{DAug}_{R}}(\mathcal{A}, \mathcal{B})$ arising from the same $R$-algebra morphisms

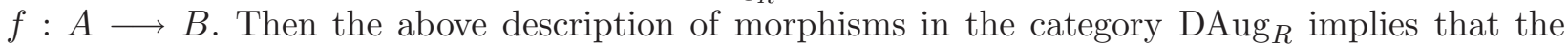
forgetful functor $\left(A, A^{b}\right) \mapsto A$ is an equivalence of $\operatorname{DAug}_{R}^{*}$ and the category of augmented finite $R$-algebras.

\subsection{Deformations of affine group schemes}

Let $\operatorname{DSch}_{R}$ be the dual category for $\operatorname{DAug}_{R}$. Its objects appear in the form $\mathcal{H}=\operatorname{Spec} \mathcal{A}=$ $\left(H, H^{b}, i_{\mathcal{H}}\right)$, where $H=\operatorname{Spec} A$ and $H^{b}=\operatorname{Spec} A^{b}$ are finite flat pointed $R$-schemes, $\mathcal{A}=\left(A, A^{b}, i_{\mathcal{A}}\right)$ $\in \operatorname{DAug}_{R}$, and $i_{\mathcal{H}}: H \rightarrow H^{\mathrm{b}}$ is a closed embedding of pointed $R$-schemes induced by $i_{\mathcal{A}}$. We agree to use the simpler notation $\left(H, H^{b}\right)$ if there is no danger of misunderstanding. The category $\operatorname{DSch}_{R}$ has direct products: if for $i=1,2, \mathcal{A}_{i}=\left(A_{i}, A_{i}^{b}, i_{\mathcal{A}_{i}}\right)$ with $A_{i}=R\left[\bar{X}_{i}\right] / I_{i}, A_{i}^{b}=R\left[\bar{X}_{i}\right] /\left(I_{i} \cdot I_{0 i}\right)$ (where $I_{0 i}=I_{R\left[\bar{X}_{i}\right]}$ ), then the product $\operatorname{Spec} \mathcal{A}_{1} \times \operatorname{Spec} \mathcal{A}_{2}$ is given by $\operatorname{Spec}\left(\mathcal{A}_{1} \otimes \mathcal{A}_{2}\right)$, where $\mathcal{A}_{1} \otimes \mathcal{A}_{2}:=\left(A_{1} \otimes_{R} A_{2},\left(A_{1} \otimes_{R} A_{2}\right)^{b}, \kappa\right),\left(A_{1} \otimes_{R} A_{2}\right)^{b}$ is the quotient of $R\left[\bar{X}_{1} \otimes 1,1 \otimes \bar{X}_{2}\right]$ by the product of ideals $I_{1} \otimes 1+1 \otimes I_{2}$ and $I_{01} \otimes 1+1 \otimes I_{02}$ and $\kappa$ is the natural projection. Note that for $i=1,2$, the projections $\operatorname{pr}_{i}: \operatorname{Spec}\left(\mathcal{A}_{1} \otimes \mathcal{A}_{2}\right) \longrightarrow \operatorname{Spec} \mathcal{A}_{i}$ come from the natural embeddings of $R\left[\bar{X}_{i}\right]$ into $R\left[\bar{X}_{1} \otimes 1,1 \otimes \bar{X}_{2}\right]$.

Let $\operatorname{DGr}_{R}$ be the category of group objects in $\operatorname{DSch}_{R}$. If $\mathcal{G}=\operatorname{Spec} \mathcal{A} \in \operatorname{DGr}_{R}$, then its group structure is given via the comultiplication $\bar{\Delta}=\left(\Delta, \Delta^{b}\right): \mathcal{A} \longrightarrow \mathcal{A} \otimes \mathcal{A}$, the counit $\bar{\varepsilon}=\left(\varepsilon, \varepsilon^{b}\right)$ : $\mathcal{A} \longrightarrow \mathcal{R}$ and the coinversion $\bar{\imath}=\left(i, i^{b}\right): \mathcal{A} \longrightarrow \mathcal{A}$ morphisms, which satisfy the usual axioms. The morphisms in $\mathrm{DGr}_{R}$ are morphisms of group objects. Clearly, $\mathrm{DGr}_{R}$ is an additive category.

Note that:

(a) $G=\operatorname{Spec} A$ is a finite flat group scheme over $R$ with the comultiplication $\Delta$, the counit $\varepsilon$ and the coinversion $i$;

(b) $\bar{\varepsilon}=\varepsilon_{\mathcal{A}}$, where $\varepsilon_{\mathcal{A}}$ is the natural augmentation from $\S 1.1$;

(c) the counit axiom gives for $i=1,2$, that $\Delta_{i}^{b} \circ \operatorname{pr}_{i}=\mathrm{id}_{A^{b}}$ and implies the uniqueness of $\Delta^{b}$ as a lifting of $\Delta$;

(d) if $\mathcal{A}=\left(A, A^{b}, i_{\mathcal{A}}\right) \in \operatorname{DAug}_{R}$ and $G=\operatorname{Spec} A$ is a finite flat group scheme over $R$, then there is a unique structure of a group object on $\operatorname{Spec} \mathcal{A}$, which is compatible with that of $G$;

(e) if $f: G \longrightarrow H$ is a morphism of group schemes and $\left(f, f^{b}\right) \in \operatorname{Hom}_{\mathrm{DSch}_{R}}(\mathcal{G}, \mathcal{H})$, then $\left(f, f^{b}\right) \in$ $\operatorname{Hom}_{\mathrm{DGr}_{R}}(\mathcal{G}, \mathcal{H})$.

The above properties have the following interpretation. Define the quotient category $\operatorname{DGr}_{R}^{*}$ as the category consisting of the objects of the category $\operatorname{DGr}_{R}$ but where $\operatorname{Hom}_{\operatorname{DGr}}^{*}(\mathcal{G}, \mathcal{H})$ consists of equivalence classes of morphisms from the category $\mathrm{DGr}_{R}$ which induce the same morphisms of group schemes $G \rightarrow H$. Then the forgetful functor $\mathcal{G} \mapsto G$ is an equivalence of categories.

\subsection{The categories of strict $O$-modules}

Suppose that $\mathcal{G}$ is an $O$-module object in the category $\operatorname{DSch}_{R}$. Then $\mathcal{G}$ is an object of $\operatorname{DGr}_{R}$ and there is a map $O \longrightarrow \operatorname{End}_{\mathrm{DGr}_{R}}(\mathcal{G})$ satisfying the usual axioms from the definition of $O$-modules. For $o \in O$ and $\mathcal{G}=\operatorname{Spec} \mathcal{A}$, denote by $[\bar{o}]=\left([o],[o]^{b}\right)$ the morphism of action of $o$ on $\mathcal{A}=\left(A, A^{b}, i_{\mathcal{A}}\right)$. Clearly, $G=\operatorname{Spec} A$ is an $O$-module in the category of finite flat schemes over $R$. For any such $G$, the $O$-module structure on the deformation $\left(G, G^{b}\right) \in \operatorname{DGr}_{R}$ is given by liftings $[o]^{b}: A^{b} \longrightarrow A^{b}$ of morphisms $[o]: A \longrightarrow A, o \in O$. Note that $[o]^{b}$ are morphisms of augmented algebras. All such liftings are automatically compatible with the group structure on this deformation, i.e. for any $o \in O$, one has $[o]^{b} \circ \Delta^{b}=\Delta^{b} \circ\left([o]^{b} \otimes[o]^{b}\right)$. So, the above system of liftings $[o]^{b}, o \in O$, gives an $O$-module 
structure if and only if for any $o_{1}, o_{2} \in O$,

$$
\left[o_{1}+o_{2}\right]^{b}=\Delta^{b} \circ\left(\left[o_{1}\right] \otimes\left[o_{2}\right]\right)^{b}, \quad\left[o_{1} o_{2}\right]^{b}=\left[o_{1}\right]^{b} \circ\left[o_{2}\right]^{b}
$$

where $\left(\left[o_{1}\right] \otimes\left[o_{2}\right]\right)^{b}$ is induced by $\left[o_{1}\right]^{b} \otimes\left[o_{2}\right]^{b}$.

Denote by $\operatorname{DGr}(O)_{R}$ the category of the above $O$-module objects where the corresponding $O$-module structure satisfies the following condition of strictness. If $\mathcal{G}=\operatorname{Spec} \mathcal{A}$ then any $o \in O$ acts on $t_{\mathcal{A}}^{*}$ and $N_{\mathcal{A}}$ via the scalar multiplication by $o$. This is the basic definition from the paper [Fal02].

Suppose that $\mathcal{G}_{1}=\left(G, G_{1}^{b}\right)=\operatorname{Spec} \mathcal{A}_{1} \in \operatorname{DGr}_{R}$ and $\mathcal{G}_{2}=\left(G, G_{2}^{b}\right)=\operatorname{Spec} \mathcal{A}_{2} \in \operatorname{DGr}_{R}$ are two deformations of a finite flat group scheme $G$ over $R$. By $\S 1.2, \mathcal{G}_{1}$ and $\mathcal{G}_{2}$ are isomorphic in the category $\mathrm{DGr}_{R}^{*}$. Suppose that $\mathcal{G}_{1}$ is equipped with a strict $O$-action. Then there is a unique (strict) $O$-action on $\mathcal{G}_{2}$ such that any $\left(\operatorname{id}_{G}, \phi\right) \in \operatorname{Hom}_{\mathrm{DGr}_{R}}\left(\mathcal{G}_{1}, \mathcal{G}_{2}\right)$ and any $\left(\operatorname{id}_{G}, \psi\right) \in \operatorname{Hom}_{\mathrm{DGr}_{R}}\left(\mathcal{G}_{2}, \mathcal{G}_{1}\right)$ are, actually, morphisms in the category $\operatorname{DGr}(O)_{R}$. This is implied by the following observation. If $\phi$ is given by the morphism of $R$-algebras $\phi^{*}: A\left(G_{2}\right)^{b} \longrightarrow A\left(G_{1}\right)^{b}$, then it induces the identifications $A\left(G_{2}\right)^{b}=\operatorname{Im} \phi^{*} \oplus J_{2}$ and $A\left(G_{1}\right)^{b}=\operatorname{Im} \phi^{*} \oplus J_{1}$, where $J_{1}, J_{2}$ are ideals such that $J_{1} \subset N_{\mathcal{A}_{1}}$ and $J_{2} \subset N_{\mathcal{A}_{2}}$.

Denote by $\operatorname{DGr}^{*}(O)_{R}$ the quotient category of $\operatorname{DGr}(O)_{R}$ where the morphisms are the equivalence classes of morphisms $\left(G, G^{b}\right) \longrightarrow\left(H, H^{b}\right)$ in the category $\operatorname{DGr}(O)_{R}$, which induce the same morphism $G \longrightarrow H$. By the above property, all isomorphism classes of objects in $\operatorname{DGr}(O)_{R}$ appear as $O$-module finite flat schemes $G$ together with a lifting of its $O$-action to some chosen deformation $G^{b}$, which satisfies the above conditions (1).

For example, if $q=p^{n}$ with $n \in \mathbb{N}$, the objects of the category $\operatorname{DGr}\left(\mathbb{F}_{q}\right)_{R}$ appear as $\operatorname{Spec} \mathcal{A}$, where $\mathcal{A}=\left(A, A^{b}\right), A=R[\bar{X}] / I$ and $A^{b}=R[\bar{X}] /\left(I \cdot I_{R[\bar{X}]}\right)$, the $\mathbb{F}_{q}$-action is induced by the scalar action of $\mathbb{F}_{q}$ on $\bar{X}$ (i.e. $[\alpha](\bar{X})=\alpha \bar{X}, \alpha \in \mathbb{F}_{q}$ ) and there are generators $j_{1}, \ldots, j_{n}$ of the ideal $I$ such that $[\alpha] j_{i}=\alpha j_{i}$ for all $i=1, \ldots, n$ and $\alpha \in \mathbb{F}_{q}$.

If $O$ is the ring of polynomials $\mathbb{F}_{q}[\pi]$ and $\mathcal{G} \in \operatorname{DGr}(O)_{R}$ then $\mathcal{G} \in \operatorname{DGr}\left(\mathbb{F}_{q}\right)_{R}$ and (in addition to the above assumptions) the $\mathbb{F}_{q}[\pi]$-action will be determined completely by the action of $\pi$ given by the correspondence

$$
\bar{X} \mapsto \psi_{\pi}(\bar{X})=\pi \bar{X}+\bar{F}(\bar{X})
$$

where $\bar{F}$ is any vector power series from $I_{R[\bar{X}]}^{2}$ such that $\bar{F}(\alpha \bar{X})=\alpha \bar{F}(\bar{X})$ for all $\alpha \in \mathbb{F}_{q}$. This action is strict if and only if $\psi_{\pi}\left(j_{i}\right) \equiv \pi j_{i} \bmod \left(I \cdot I_{R[\bar{X}]}\right)$ for the above generators $j_{1}, \ldots, j_{n}$ of $I$.

Finally, note that when classifying below the objects $\left(G, G^{b}\right)$ of the categories $\operatorname{DGr}\left(\mathbb{F}_{q}\right)_{R}$ and $\operatorname{DGr}\left(\mathbb{F}_{q}[\pi]\right)_{R}$ we use a choice of the deformation $G^{b}$ that depends functorially on $G$.

\section{Group schemes with strict $\mathbb{F}_{q \text {-action }}$}

In this section we assume that $R$ is an $\mathbb{F}_{p}$-algebra and study the category $\operatorname{DGr}\left(\mathbb{F}_{p}\right)_{R}$ of strict finite $\mathbb{F}_{p}$-modules $G$ over $R$. Clearly, $\operatorname{DGr}(\mathbb{Z})_{R}=\operatorname{DGr}_{R}$, i.e. the natural action of $\mathbb{Z}$ on elements of $\operatorname{DGr}_{R}$ is always strict. Therefore, $\left(G, G^{b}\right) \in \operatorname{DGr}\left(\mathbb{F}_{p}\right)_{R}$ if and only if $\left(G, G^{b}\right) \in \operatorname{DGr}_{R}$ and $[p]^{b}$ is a zero map on $G^{b}$. In $\S \S 2.1$ and 2.2 we give a complete description of the category $\operatorname{DGr}\left(\mathbb{F}_{p}\right)_{R}$ and in $\S 2.3$ apply it to describe the category $\operatorname{DGr}\left(\mathbb{F}_{q}\right)_{R}$, where $q=p^{N}$ with $N \in \mathbb{N}$.

\subsection{An interpretation of strict $\mathbb{F}_{\boldsymbol{p}}$-action}

Suppose that $G$ is a finite flat commutative group scheme over $R$. Consider $G^{(p)}=G \times_{\left(R, \sigma_{p}\right)} R$, where $R$ is considered as an $R$-module via the map $\sigma_{p}: R \longrightarrow R$ such that for any $r \in R, \sigma_{p}(r)=r^{p}$. Then $G^{(p)}$ has a natural structure of a finite flat commutative group scheme over $R$.

Let $F_{G}: G \longrightarrow G^{(p)}$ be the relative Frobenius morphism of $G$ over Spec $R$. It is given by the morphism of $R$-algebras $F_{G}^{*}: A\left(G^{(p)}\right)=A(G) \otimes_{\left(R, \sigma_{p}\right)} R \longrightarrow A(G)$ such that for all $a \in A(G)$ and $r \in R, F_{G}^{*}(a \otimes r)=a^{p} r$. 


\section{Abrashikin}

Let $V_{G}: G^{(p)} \longrightarrow G$ be the Verschiebung morphism of group schemes. Recall that it is given by the morphism of $R$-algebras $V_{G}^{*}: A(G) \longrightarrow A\left(G^{(p)}\right)$, which can be described as follows. We can proceed locally on $\operatorname{Spec} R$ and, therefore, can assume that $A(G)$ is a free $R$-module with a basis $a_{1}, \ldots, a_{n}$. Let

$$
\Delta^{(p)}=\Delta \circ(\Delta \otimes \mathrm{id}) \circ \cdots \circ\left(\Delta \otimes \mathrm{id}^{\otimes p-2}\right): A(G) \longrightarrow A(G)^{\otimes p} .
$$

For $1 \leqslant i \leqslant n$, we have a unique decomposition

$$
\Delta^{(p)}\left(a_{i}\right)=\sum_{1 \leqslant i_{1}, \ldots, i_{p} \leqslant n} \alpha_{i, i_{1}, \ldots, i_{p}} a_{i_{1}} \otimes \cdots \otimes a_{i_{p}}
$$

with all $\alpha$-coefficients from $R$. Then the Verschiebung is defined by the relation

$$
V_{G}^{*}\left(a_{i}\right)=\sum_{1 \leqslant j \leqslant n} \alpha_{i, j, \ldots, j} \otimes a_{j} .
$$

The above definitions imply easily that $F_{G} \circ V_{G}=p \operatorname{id}_{G}$ and $V_{G} \circ F_{G}=p \operatorname{id}_{G(p)}$, cf. our agreement about composition of maps from $\S 0$.

Theorem 1. We have $\mathcal{G}=\left(G, G^{b}\right) \in \operatorname{DGr}\left(\mathbb{F}_{p}\right)_{R}$ if and only if $V_{G}=0$.

Proof. Both properties can be verified locally on $\operatorname{Spec} R$. Therefore, we can assume that $R$ is a local ring. As we have already noticed, $\mathcal{G} \in \operatorname{DGr}\left(\mathbb{F}_{p}\right)_{R}$ if and only if $[p]^{b}$ is a zero morphism.

2.1.1 Suppose first that $V_{G}=0$. Use the antiequivalence $\mathrm{L}_{p}$ of the category of finite flat commutative group schemes $G$ over $R$ with zero Verschiebung and the category $\operatorname{Mod}\left(\mathbb{F}_{p}\right)_{R}$ of locally free finite $R$-modules $L$ equipped with an $R$-linear map $F_{p}: L^{(p)}:=L \otimes_{R, \sigma_{p}} R \longrightarrow L$, cf. [Gab70, $\left.\mathrm{VII}_{\mathrm{A}}, 7.4\right]$. Here $\mathrm{L}_{p}(G)=\left(L, F_{p}\right)$, where

$$
L=\operatorname{Hom}\left(G, \mathbb{G}_{a}\right)=\{a \in A(G) \mid \Delta(a)=a \otimes 1+1 \otimes a\}
$$

is a free $R$-module of finite $R$-rank and $F_{p}$ is induced by the Frobenius $F_{G}^{*}$.

The inverse functor $\mathrm{D}_{p}$ can be described as follows. If $\mathcal{L}=\left(L, F_{p}\right) \in \operatorname{Mod}\left(\mathbb{F}_{p}\right)_{R}$ and $m_{1}, \ldots, m_{n}$ is an $R$-basis for $L$ then for $1 \leqslant i \leqslant n$,

$$
F_{p}\left(m_{i} \otimes 1\right)=\sum_{1 \leqslant j \leqslant n} r_{i j} m_{j} .
$$

Then $\mathrm{D}_{p}(\mathcal{L})=\operatorname{Spec} A(G)$, where $A(G)=R\left[X_{1}, \ldots, X_{n}\right] / I$ with the ideal $I$ generated by the polynomials

$$
X_{i}^{p}-\sum_{1 \leqslant j \leqslant n} r_{i j} X_{j}, \quad 1 \leqslant i \leqslant n,
$$

with the group structure given via the comultiplication $\Delta$ such that $\Delta\left(X_{i}\right)=X_{i} \otimes 1+1 \otimes X_{i}$ and the counit $e$ such that $e\left(X_{i}\right)=0$ for $1 \leqslant i \leqslant n$. Note that $A(G)$ is a finite flat $R$-algebra, given by $n$ equations (2) in $R\left[X_{1}, \ldots, X_{n}\right]$ and, therefore, it is a relative complete intersection.

With the above notation take $G^{b}=\operatorname{Spec} A(G)^{b}$, where $A(G)^{b}=R\left[X_{1}, \ldots, X_{n}\right] /\left(I \cdot I_{0}\right)$ with $I_{0}=\left(X_{1}, \ldots, X_{n}\right)$. Note that the $R$-module $A(G)^{b}$ is free with the basis

$$
\left\{X_{1}^{i_{1}} \ldots X_{n}^{i_{n}} \mid 0 \leqslant i_{1}, \ldots, i_{n}<p\right\} \cup\left\{X_{i}^{p} \mid 1 \leqslant i \leqslant n\right\} .
$$

Similarly, $A(G \times G)^{b}$ is a free $R$-module with the basis

$$
\left\{X_{1}^{i_{1}} \ldots X_{n}^{i_{n}} \otimes X_{1}^{j_{1}} \ldots X_{n}^{j_{n}} \mid 0 \leqslant i_{1}, \ldots, i_{n}, j_{1}, \ldots, j_{n}<p\right\} \cup\left\{X_{i}^{p} \otimes 1,1 \otimes X_{i}^{p} \mid 1 \leqslant i \leqslant n\right\} .
$$

With this notation we have $e^{b}\left(X_{i}\right)=0$ and

$$
\Delta^{b}\left(X_{i}\right)=X_{i} \otimes 1+1 \otimes X_{i}+\sum_{1 \leqslant j \leqslant n}\left(r_{i j}^{\prime} X_{j}^{p} \otimes 1+r_{i j}^{\prime \prime} 1 \otimes X_{j}^{p}\right)
$$




\section{FALTINGS'S STRICT MODULES}

with $r$-coefficients from $R$. Then the identities

$$
\Delta^{b} \circ\left(\operatorname{id}_{A(G)} \otimes e\right)^{b}=\Delta^{b} \circ\left(e \otimes \operatorname{id}_{A(G)}\right)^{b}=\operatorname{id}_{A(G)^{b}}
$$

imply that all $r$-coefficients are equal to 0 . This easily implies that

$$
[p]^{b}=\Delta^{(p) b} \circ \operatorname{mult}_{p}^{b}=0,
$$

where $\operatorname{mult}_{p}^{b}: A(G)^{\otimes p b} \longrightarrow A(G)^{b}$ is induced by the multiplication in $A(G)^{b}$.

2.1.2 Suppose now that $\mathcal{G}=\left(G, G^{b}\right) \in \operatorname{DGr}\left(\mathbb{F}_{p}\right)_{R}$. When proving that this implies $V_{G}=0$ we can use any flat base changes. Therefore, we can assume that $R$ is a finitely generated complete local ring with an algebraically closed residue field $k$ of characteristic $p$. Then $R$ is an inverse limit of artinian rings and by transfinite induction it will be sufficient to consider the following two cases:

(a) $R=k$;

(b) $R$ contains an ideal $J$ such that $J \cdot I_{R}=0$ (where $I_{R}$ is the augmentation ideal in $R$ ) and if $\bar{R}=R / J$ and $\bar{G}=G \otimes_{R} \bar{R}$, then $V_{\bar{G}}=0$.

2.1.3 Case (a). Apply induction on the order $|G|=p^{N}, N \in \mathbb{N}$. If $N=1$, then there are the following three possibilities:

- $G \simeq(\mathbb{Z} / p \mathbb{Z})_{/ k}$, i.e. it is the constant etale group scheme of order $p$;

- $G \simeq \alpha_{p}$, i.e. $A(G)=k[x], x^{p}=0, \Delta(x)=x \otimes 1+1 \otimes x$;

- $G \simeq \mu_{p}$, i.e. it is the constant multiplicative group scheme of order $p$.

In the first two cases $V_{G}=0$. In the third case $V_{G}=\mathrm{id}_{G}$, but $[p]^{\mathrm{b}} \neq 0$. Indeed, $A\left(\mu_{p}\right)^{b}=$ $k[x] /\left(x^{p+1}\right), e^{b}(x)=0, \Delta^{b}(1+x)=(1+x) \otimes(1+x)$ and $[p]^{b}(1+x)=(1+x)^{p}=1+x^{p} \neq 1=e^{b}(1+x)$ in $A\left(\mu_{p}\right)^{b}$.

Now assume that $N>1$ and $V_{G_{1}}=0$ for any proper subquotient of $G$. Consider the Dieudonne module $M(G)=\operatorname{Hom}\left(G, C W_{k}\right)$ of $G$, where $C W_{k}$ is the $k$-valued Witt covectors functor, cf. [Fon75]. Then $M(G)$ is a $k$-vector space of dimension $N$ with a $\sigma_{p}$-linear operator $F$ and a $\sigma_{p}^{-1}$-linear operator $V$, which are induced by $F_{G}$ and $V_{G}$, respectively. The inductive assumption implies the existence of a $k$-basis $m_{1}, \ldots, m_{N}$ in $M(G)$ such that for $1 \leqslant i \leqslant N, F m_{i}=\sum_{1 \leqslant i \leqslant N} c_{i j} m_{j}$, $V m_{1}=\cdots=V m_{N-1}=0$ and $V m_{N}=c_{0} m_{1}$ where all $c$-coefficients belong to $k$. By the Dieudonne theory,

$$
A(G)=k\left[X_{1}, \ldots, X_{N}\right] / I,
$$

where the ideal $I$ is generated by the polynomials $X_{i}^{p}-\sum c_{i j} X_{j}, 1 \leqslant i \leqslant N$, with the counit $e: A(G) \longrightarrow k$ such that $e\left(X_{i}\right)=0,1 \leqslant i \leqslant N$, and the comultiplication $\Delta: A(G) \longrightarrow A(G) \otimes A(G)$ such that for $1 \leqslant i<N, \Delta\left(X_{i}\right)=X_{i} \otimes 1+1 \otimes X_{i}$ and

$$
\Delta\left(X_{N}\right)=X_{N} \otimes 1+1 \otimes X_{N}+c_{0} \varphi\left(X_{1} \otimes 1,1 \otimes X_{1}\right) .
$$

Here $\varphi(T, U) \in k[T, U]$ is the polynomial $\frac{1}{p}\left(T^{p}+U^{p}-(T+U)^{p}\right) \in \mathbb{Z}[T, U]$ taken modulo $p$.

As earlier, take $A(G)^{b}=k\left[X_{1}, \ldots, X_{N}\right] /\left(I \cdot I_{0}\right)$ with $I_{0}=\left(X_{1}, \ldots, X_{N}\right)$. Consider the $k$-basis

$$
\left\{X_{1}^{i_{1}} \ldots X_{N}^{i_{N}} \mid 0 \leqslant i_{1}, \ldots, i_{N}<p\right\} \cup\left\{X_{i}^{p} \mid 1 \leqslant i \leqslant N\right\}
$$

in $A(G)^{b}$ and the $k$-basis

$$
\left\{X_{1}^{i_{1}} \ldots X_{N}^{i_{N}} \otimes X_{1}^{j_{1}} \ldots X_{N}^{j_{N}} \mid 0 \leqslant i_{s}, j_{s}<p, 1 \leqslant s \leqslant N\right\} \cup\left\{X_{i}^{p} \otimes 1,1 \otimes X_{i}^{p} \mid 1 \leqslant i \leqslant N\right\}
$$

in $A(G \times G)^{b}$. Similarly to $\S 2.1 .1$ one sees that

$$
\Delta^{b}\left(X_{N}\right)=X_{N} \otimes 1+1 \otimes X_{N}+c_{0} \varphi\left(X_{1} \otimes 1,1 \otimes X_{1}\right)
$$




\section{ABRASHKIN}

and $\Delta^{b}\left(X_{1}\right)=X_{1} \otimes 1+1 \otimes X_{1}$. This implies easily that

$$
\begin{aligned}
\Delta^{(p) b}\left(X_{N}\right)= & X_{N} \otimes 1 \otimes \cdots \otimes 1+1 \otimes X_{N} \otimes 1 \otimes \cdots \otimes 1+\cdots+1 \otimes \cdots \otimes 1 \otimes X_{N} \\
& +c_{0} \varphi^{(p)}\left(X_{1} \otimes 1 \otimes \cdots \otimes 1,1 \otimes X_{1} \otimes 1 \otimes \cdots \otimes 1, \ldots, 1 \otimes \cdots \otimes 1 \otimes X_{1}\right),
\end{aligned}
$$

where $\varphi^{(p)}\left(U_{1}, \ldots, U_{p}\right) \in k\left[U_{1}, \ldots, U_{p}\right]$ is the reduction of the polynomial

$$
\frac{1}{p}\left(U_{1}^{p}+\cdots+U_{p}^{p}-\left(U_{1}+\cdots+U_{p}\right)^{p}\right) \in \mathbb{Z}\left[U_{1}, \ldots, U_{p}\right]
$$

modulo $p$. Therefore, $0=[p]^{b}\left(X_{N}\right)=\left(\Delta^{(p)} \circ \text { mult }_{p}\right)^{b}\left(X_{N}\right)=c_{0} X_{1}^{p}$, hence $c_{0}=0$ and $V_{G}=0$. Case (a) is completely considered.

Remark. Case (a) was also considered in [Gib04].

2.1.4 Case (b). Since $V_{\bar{G}}=0$, we can again apply the antiequivalence from [Gab70], to describe explicitly the structure of $\bar{G}$. It can be given by the algebra $A(\bar{G})=\bar{R}\left[\bar{X}_{1}, \ldots, \bar{X}_{N}\right] / \bar{I}$, where $\bar{I}$ is generated by the polynomials

$$
\bar{X}_{i}^{p}-\sum_{1 \leqslant j \leqslant N} \bar{c}_{i j} \bar{X}_{j} \in \bar{R}\left[\bar{X}_{1}, \ldots, \bar{X}_{N}\right]
$$

with the comultiplication $\Delta\left(\bar{X}_{i}\right)=\bar{X}_{i} \otimes 1+1 \otimes \bar{X}_{i}, 1 \leqslant i \leqslant N$.

Let $l_{1}, \ldots, l_{s}$ be a $k$-basis of the ideal $J$ with respect to its natural structure as a $k\left(=R / I_{R}\right)$ module. As $A(G)$ is a flat $R$-module we can take liftings $X_{i} \in A(G)$ of $\bar{X}_{i}$ and $c_{i j} \in R$ of $\bar{c}_{i j} \in \bar{R}$ such that $A(G)=R\left[X_{1}, \ldots, X_{N}\right] / I$, where $I$ is generated by the polynomials of the form

$$
X_{i}^{p}-\sum_{1 \leqslant j \leqslant N} c_{i j} X_{j}-\sum_{1 \leqslant t \leqslant s} f_{i t} l_{t}, \quad 1 \leqslant i \leqslant N,
$$

with $f_{i t} \in A(G) \otimes J \simeq A\left(G_{k}\right)$ and $G_{k}=G \otimes k$.

Similarly,

$$
\Delta\left(X_{i}\right)=X_{i} \otimes 1+1 \otimes X_{i}+\sum_{1 \leqslant t \leqslant s} g_{i t} l_{t}, \quad 1 \leqslant i \leqslant N,
$$

where all $g_{i t} \in A\left(G_{k} \times G_{k}\right)$.

Consider the second Hochschild cohomology group $H^{2}(G, k)$. Recall that it appears as the quotient $Z^{2}(G, k) / B^{2}(G, k)$, where

$$
\begin{gathered}
Z^{2}(G, k)=\left\{f \in A\left(G_{k} \times G_{k}\right) \mid(\Delta \otimes \mathrm{id})(f)+f \otimes 1=1 \otimes f+(\mathrm{id} \otimes \Delta)(f)\right\}, \\
B^{2}(G, k)=\left\{\Delta(h)-h \otimes 1-1 \otimes h \mid h \in A\left(G_{k}\right)\right\} .
\end{gathered}
$$

As $V_{G_{k}}=0, H^{2}(G, k)$ is an $N$-dimensional $k$-vector space with the basis given by the classes of 2-cocycles $\varphi\left(X_{i} \otimes 1,1 \otimes X_{i}\right), 1 \leqslant i \leqslant N$, cf. [DG70]. This implies that the liftings $X_{i} \in A(G)$ of $\bar{X}_{i} \in A(\bar{G})$ can be chosen such that in the equalities $(3)$ for $1 \leqslant i \leqslant N$ and $1 \leqslant t \leqslant s$,

$$
g_{i t}=\sum_{1 \leqslant j \leqslant N} \alpha_{i j t} \varphi\left(X_{j} \otimes 1,1 \otimes X_{j}\right),
$$

where all $\alpha$-coefficients belong to $k$.

As earlier, this implies that

$$
\begin{aligned}
\Delta^{(p)}\left(X_{i}\right)= & X_{i} \otimes 1 \otimes \cdots \otimes 1+1 \otimes X_{i} \otimes 1 \otimes \cdots \otimes 1+\cdots+1 \otimes \cdots \otimes 1 \otimes X_{i} \\
& +\sum_{j, t} \alpha_{i j t} l_{t} \varphi^{(p)}\left(X_{j} \otimes 1 \otimes \cdots \otimes 1,1 \otimes X_{j} \otimes 1 \otimes \cdots \otimes 1, \ldots, 1 \otimes \cdots \otimes 1 \otimes X_{j}\right)
\end{aligned}
$$


and, therefore, for all $1 \leqslant i \leqslant N$,

$$
V_{G}\left(X_{i}\right)=\sum_{j, t} \alpha_{i j t} l_{t} X_{j}
$$

On the other hand, we have (as earlier)

$$
\Delta^{b}\left(X_{i}\right)=X_{i} \otimes 1+1 \otimes X_{i}+\sum_{j, t} \alpha_{i j t} l_{t} \varphi\left(X_{j} \otimes 1,1 \otimes X_{j}\right)
$$

in $A(G)^{b}=R\left[X_{1}, \ldots, X_{N}\right] /\left(I \cdot I_{0}\right)$ and this implies that

$$
0=[p]^{b}\left(X_{i}\right)=\sum_{j, t} \alpha_{i j t} l_{t} X_{j}^{p} .
$$

Therefore, all $\alpha$-coefficients are equal to 0 and $V_{G}=0$.

The theorem is completely proved.

The above Theorem 1 together with the antiequivalence $\mathrm{L}_{p}$ from the beginning of $\S 2.1 .1$ means that the forgetful functor $\mathcal{G}=\left(G, G^{b}\right) \mapsto G$ is an equivalence of the category of group schemes over $R$ with strict $\mathbb{F}_{p}$-action $\operatorname{DGr}\left(\mathbb{F}_{p}\right)_{R}$ and the full subcategory of the category of finite flat group schemes $G$ over $R$ such that $V_{G}=0$. The resulting antiequivalence of the categories $\operatorname{DGr}\left(\mathbb{F}_{p}\right)_{R}$ and $\operatorname{Mod}\left(\mathbb{F}_{p}\right)_{R}$ will be denoted by the same symbol $\mathrm{L}_{p}$.

\subsection{Standard deformations of strict $\mathbb{F}_{p}$-modules}

By Theorem 1 the objects $\mathcal{G} \in \operatorname{DGr}\left(\mathbb{F}_{p}\right)_{R}$ are constructed from locally free $R$-modules $L$ of finite $R$-rank with a given $R$-linear map $F_{p}: L^{(p)} \longrightarrow L$. This construction gives $\mathcal{G}=\operatorname{Spec} \mathcal{A}$ with $\mathcal{A}=$ $\left(A(L), A(L)^{b}, i_{\mathcal{A}}\right)$, where $A(L)=\operatorname{Sym}_{R}(L) / I, A(L)^{b}=\operatorname{Sym}_{R}(L) /\left(I \cdot I_{0}\right)$, the ideal $I$ is generated by $\left\{l^{p}-F_{p}(l \otimes 1) \mid l \in L\right\}, I_{0}$ is the augmentation ideal generated by $\{l \mid l \in L\}$ and the comultiplications $\Delta$ and $\Delta^{b}$ are induced by the correspondences $l \mapsto l \otimes 1+1 \otimes l$. This deformation $\mathcal{G}$ depends functorially on the group scheme $G=\operatorname{Spec} A(L)$. Note that $L=\{a \in A(L) \mid \Delta(a)=a \otimes 1+1 \otimes a\}$.

Definition. With the above notation, set $L^{b}=\left\{l \in A(L)^{b} \mid \Delta^{b}(l)=l \otimes 1+1 \otimes l\right\}$.

Such $L^{b}$ is a locally free $R$-module of $\operatorname{rank} 2 \operatorname{rk}_{R} L$. In the notation of $\S 2.1 .1$ it is generated as an $R$-module by the elements $X_{1}, \ldots, X_{n}, X_{1}^{p}, \ldots, X_{n}^{p}$ in the algebra $A(L)^{b}$. Clearly, we have a natural projection $i_{\mathcal{A}}: L^{b} \longrightarrow L$. There is also a natural inclusion

$$
N_{\mathcal{A}}=\left\{l^{p}-F_{p}(l \otimes 1) \mid l \in L\right\} \bmod \left(I \cdot I_{0}\right) \subset L^{b},
$$

the natural projection $\mathrm{pr}: L^{b} \longrightarrow t_{\mathcal{A}}^{*}$ given by the correspondence $l \mapsto l \bmod I_{0}^{2}$, where $l \in L$, and the $R$-linear map $j_{\mathcal{A}}: L^{(p)} \longrightarrow L^{b}$ induced by the correspondence $l \mapsto \hat{l}^{p}$, where $\hat{l} \in L^{b}$ is such that $i_{\mathcal{A}}(\hat{l})=l$.

The following lemma is an easy consequence of the above definitions.

LEMma. There are exact sequences of locally free $R$-modules

$$
0 \longrightarrow N_{\mathcal{A}} \longrightarrow L^{b} \stackrel{i_{\mathcal{A}}}{\longrightarrow} L \longrightarrow 0
$$

and

$$
0 \longrightarrow L^{(p)} \stackrel{j_{\mathcal{A}}}{\longrightarrow} L^{b} \longrightarrow t_{\mathcal{A}}^{*} \longrightarrow 0 .
$$

\subsection{Group schemes with strict $\mathbb{F}_{q^{-} \text {-action }}$}

Assume now that $R$ is an $\mathbb{F}_{q}$-algebra, where $q=p^{N}$ and $N \in \mathbb{N}$, and study the category $\operatorname{DGr}\left(\mathbb{F}_{q}\right)_{R}$ of strict finite $\mathbb{F}_{q}$-modules $G$ over $R$ as a full subcategory of $\operatorname{DGr}\left(\mathbb{F}_{p}\right)_{R}$. 


\section{Abrashikin}

For any $R$-module $M$ set $M^{(q)}=M \otimes_{\left(R, \sigma_{q}\right)} R$, where the $R$-module structure on the second component of this tensor product is given via the $q$ th power map $\sigma_{q}: R \longrightarrow R$. If $f: M \longrightarrow N$ is a morphism of $R$-modules, then we use the notation $f^{(q)}=f \otimes_{\left(R, \sigma_{q}\right)} R: M^{(q)} \longrightarrow N^{(q)}$.

Consider the category $\operatorname{Mod}\left(\mathbb{F}_{q}\right)_{R}$ consisting of locally free finite rank $R$-modules $M$ with an $R$-linear morphism $F_{q}: M^{(q)} \longrightarrow M$. If $N$ is another object, then $\operatorname{Hom}_{\operatorname{Mod}\left(\mathbb{F}_{q}\right)_{R}}(M, N)$ consists of $R$-linear morphisms $f: M \longrightarrow N$ such that $F_{q} \circ f=f^{(q)} \circ F_{q}$.

Define the functor $\mathrm{L}_{q}: \operatorname{DGr}\left(\mathbb{F}_{q}\right)_{R} \longrightarrow \operatorname{Mod}\left(\mathbb{F}_{q}\right)_{R}$ by setting for any $\mathcal{G}=\left(G, G^{b}\right) \in \operatorname{DGr}\left(\mathbb{F}_{q}\right)_{R}$, $\mathrm{L}_{q}(\mathcal{G})=\left(L(\mathcal{G}), F_{q}\right)$ with

$$
L(\mathcal{G})=\left\{a \in A(G) \mid \Delta(a)=a \otimes 1+1 \otimes a,[\alpha](a)=\alpha a, \forall \alpha \in \mathbb{F}_{q}\right\},
$$

where $F_{q}: L(\mathcal{G})^{(q)} \longrightarrow L(\mathcal{G})$ is induced by the $q$ th power map on $A(G)$. If $\mathcal{H} \in \operatorname{DGr}\left(\mathbb{F}_{q}\right)_{R}, \mathrm{~L}_{q}(\mathcal{H})=$ $\left(L(\mathcal{H}), F_{q}\right)$ and $\left(f, f^{b}\right): \mathcal{G} \longrightarrow \mathcal{H}$ is a morphism in the category $\operatorname{DGr}\left(\mathbb{F}_{q}\right)_{R}$, then $f(L(\mathcal{H})) \subset L(\mathcal{G})$ and $\mathrm{L}_{q}(f)=\left.f\right|_{L(\mathcal{H})}$.

Theorem 2. The above defined functor $\mathrm{L}_{q}: \operatorname{DGr}\left(\mathbb{F}_{q}\right)_{R} \longrightarrow \operatorname{Mod}\left(\mathbb{F}_{q}\right)_{R}$ induces an antiequivalence of the categories $\operatorname{DGr}^{*}\left(\mathbb{F}_{q}\right)_{R}$ and $\operatorname{Mod}\left(\mathbb{F}_{q}\right)_{R}$.

Proof. The proof below is standard: cf., for example, [Ray74] (in the context of finite flat group schemes with $\mathbb{F}_{q}$-action) or [Gen96] (in the context of formal modules).

2.3.1 We first construct the functor $\mathrm{D}_{q}: \operatorname{Mod}\left(\mathbb{F}_{q}\right)_{R} \longrightarrow \operatorname{DGr}^{*}\left(\mathbb{F}_{q}\right)_{R}$ in a similar manner to the construction from $\S 2.1 .1$.

If $\left(L, F_{q}\right) \in \operatorname{Mod}\left(\mathbb{F}_{q}\right)_{R}$, then $\mathrm{D}_{q}\left(L, F_{q}\right)=\operatorname{Spec} \mathcal{A}$ with $\mathcal{A}=\left(A(G), A(G)^{b}, i_{\mathcal{A}}\right)$, defined by:

- $A(G)=\operatorname{Sym}_{R}(L) / I$ where the ideal $I$ is generated by $\left\{l^{q}-F_{q}(l \otimes 1) \mid l \in L\right\}$, the comultiplication $\Delta$ is such that $\Delta(l)=l \otimes 1+1 \otimes l$ and the $\mathbb{F}_{q}$-action is such that $[\alpha](l)=\alpha l$ for all $l \in L$ and $\alpha \in \mathbb{F}_{q}$;

- $A(G)^{b}=\operatorname{Sym}_{R}(L) /\left(I \cdot I_{0}\right)$ where the augmentation ideal $I_{0}$ is generated by all $l \in L$, the comultiplication $\Delta^{b}$ is such that $\Delta^{b}(l)=l \otimes 1+1 \otimes l$ and the $\mathbb{F}_{q}$-action $[\alpha]^{b}$ is given by the correspondences $l \mapsto l \otimes 1+1 \otimes l$ and $l \mapsto \alpha l$ for all $l \in L$ and $\alpha \in \mathbb{F}_{q}$;

- $i_{\mathcal{A}}$ is the natural projection from $A(G)^{b}$ to $A(G)$.

Clearly, $\mathcal{G}=\operatorname{Spec} \mathcal{A} \in \operatorname{DGr}\left(\mathbb{F}_{q}\right)_{R}$ and the correspondence $\mathrm{D}_{q}:\left(L, F_{q}\right) \mapsto \mathcal{G}$ can be naturally extended to the functor $\mathrm{D}_{q}: \operatorname{Mod}\left(\mathbb{F}_{q}\right)_{R} \longrightarrow \operatorname{DGr}\left(\mathbb{F}_{q}\right)_{R}$. This functor is additive and faithful.

2.3.2 We now prove that any $\mathcal{G} \in \operatorname{DGr}\left(\mathbb{F}_{q}\right)_{R}$ can be identified in the category $\operatorname{DGr}^{*}\left(\mathbb{F}_{q}\right)_{R}$ with some $\mathrm{D}_{q}\left(L_{0}, F_{q}\right)$, where $\left(L_{0}, F_{q}\right) \in \operatorname{Mod}\left(\mathbb{F}_{q}\right)_{R}$. Since $\mathcal{G} \in \operatorname{DGr}\left(\mathbb{F}_{p}\right)_{R}$ we can assume that it is presented by the deformation $\operatorname{Spec}\left(A, A^{b}\right)$ where the $R$-algebras $A$ and $A^{b}$ are described in terms of the $R$-module $L$ such that $\left(L, F_{p}\right)=\mathrm{L}_{p}(\mathcal{G})$, cf. $\S 2.2$.

For all $\alpha \in \mathbb{F}_{q}$, there are the $R$-linear actions $[\alpha]: L \longrightarrow L$ and $[\alpha]^{b}: L^{b} \longrightarrow L^{b}$. Therefore, we have the direct sum decompositions of locally free modules $L=\bigoplus_{n \in \mathbb{Z} / N \mathbb{Z}} L_{n}$ and $L^{b}=\bigoplus_{n \in \mathbb{Z} / N \mathbb{Z}} L_{n}^{b}$, where for all $n \in \mathbb{Z} \bmod N$,

$$
L_{n}=\left\{l \in L \mid[\alpha](l)=\sigma_{p}^{n}(\alpha) l, \forall \alpha \in \mathbb{F}_{q}\right\}, \quad L_{n}^{b}=\left\{l \in L^{b} \mid[\alpha](l)=\sigma_{p}^{n}(\alpha) l, \forall \alpha \in \mathbb{F}_{q}\right\} .
$$

The exact sequence (4) from the lemma in $\S 2.2$ implies that $i_{\mathcal{A}}$ induces isomorphisms of $R$-modules $i_{n}: L_{n}^{b} \longrightarrow L_{n}$ for all $n \in \mathbb{Z} / N \mathbb{Z}, n \neq 0$. Similarly, exact sequence (5) from the same lemma implies that $j_{\mathcal{A}}$ induces isomorphisms of $R$-modules $j_{n}: L_{n-1} \longrightarrow L_{n}^{b}$ for all $n \in \mathbb{Z} / N \mathbb{Z}$, $n \neq 0$. Then from the relation $j_{\mathcal{A}} \circ i_{\mathcal{A}}=F_{p}$ it follows that for all $n=0, \ldots, N-2, F_{p}$ induces isomorphisms $L_{n} \simeq L_{n+1}$ and, therefore, $L=\bigoplus_{n \in \mathbb{Z} \bmod N} F_{p}^{n}\left(L_{0}\right)$. 


\section{FALTINGS'S STRICT MODULES}

Let $\kappa: \operatorname{Sym}_{R}(L) \longrightarrow \operatorname{Sym}_{R}\left(L_{0}\right)$ be an $R$-algebra morphism uniquely determined by the correspondences $\kappa: F_{p}^{n}\left(l_{0}\right) \mapsto l_{0}^{p^{n}}$ for all $0 \leqslant n<N$ and $l_{0} \in L_{0}$. Then a straightforward calculation shows that $\kappa$ induces an isomorphism of $\mathcal{G}$ and $\mathrm{D}_{q}\left(L_{0}, F_{q}\right)$ in the category $\operatorname{DGr}^{*}\left(\mathbb{F}_{q}\right)_{R}$. The theorem is proved.

\section{Group schemes with strict $\mathbb{F}_{q}[\pi]$-action}

Suppose that $O=\mathbb{F}_{q}[\pi]$ with a fixed indeterminate $\pi$ and $\mathcal{G}=\operatorname{Spec} \mathcal{A}(\mathcal{G}) \in \operatorname{DGr}(O)_{R}$.

\subsection{The $R$-module $L(\mathcal{G})^{b}$}

Consider $\mathcal{G}$ as an object of the category $\operatorname{DGr}\left(\mathbb{F}_{q}\right)_{R}$. Then there is a $\left(L, F_{q}\right) \in \operatorname{Mod}\left(\mathbb{F}_{q}\right)_{R}$ such that $\mathcal{G}$ is isomorphic to $\operatorname{Spec} \mathcal{A}$, where $\mathcal{A}=\left(A(G), A(G)^{b}, i_{\mathcal{A}}\right)$ is given in the notation of $\S 2.3 .1$.

Note that $L=L(\mathcal{G})=\left\{a \in A(G) \mid \Delta(a)=a \otimes 1+1 \otimes a,[\alpha](a)=\alpha a, \forall \alpha \in \mathbb{F}_{q}\right\}$. Similarly to $\S 2.2$ introduce

$$
L^{b}=L(\mathcal{G})^{b}=\left\{a \in A(G)^{b} \mid \Delta^{b}(a)=a \otimes 1+1 \otimes a,[\alpha](a)=\alpha a, \forall \alpha \in \mathbb{F}_{q}\right\} .
$$

Then $L^{b}$ is a locally free $R$-module, $\operatorname{rk}_{R} L^{b}=2 \mathrm{rk}_{R} L$, it is generated by the images of elements $\left\{l, l^{q} \mid l \in L\right\}$ in $A(G)^{b}$ and therefore can be identified with $L \oplus L^{(q)}$.

The action $[\pi]_{\mathcal{G}}=\left([\pi],[\pi]^{b}\right)$ of $\pi \in O$ on $\mathcal{G}$ is uniquely determined by $R$-linear endomorphisms $[\pi]: L \longrightarrow L$ and $[\pi]^{b}: L^{b} \longrightarrow L^{b}$ such that $[\pi]^{b} \circ i_{L}=i_{L} \circ[\pi]$, where $i_{L}=\left.i_{\mathcal{A}}\right|_{L^{b}}: L^{b} \longrightarrow L$ is an epimorphism of $R$-modules.

Note that $N_{\mathcal{A}}=I \bmod \left(I \cdot I_{0}\right) \subset L^{b}$. With respect to the above-mentioned identification $L^{b}=$ $L \oplus L^{(q)}$, we have $i_{L}\left(l_{1}, l_{2}\right)=l_{1}+F_{q}\left(l_{2}\right), N_{\mathcal{A}}=\left\{\left(F_{q}(l),-l\right) \mid l \in L^{(q)}\right\}$ and the natural sequence

$$
0 \longrightarrow N_{\mathcal{A}} \longrightarrow L^{b} \stackrel{i_{L}}{\longrightarrow} L \longrightarrow 0
$$

is an exact sequence of $O-R$-modules.

Consider the $R$-linear morphism $j_{L}: L^{(q)} \longrightarrow L^{b}$ given by the correspondence $l \otimes 1 \mapsto \hat{l}^{q}$, where $l \in L$ and $\hat{l} \in L^{b}$ is such that $i_{L}(\hat{l})=l$. Clearly, $j_{L} \circ i_{L}=F_{q}$ and the sequence

$$
0 \longrightarrow L^{(q)} \stackrel{j_{L}}{\longrightarrow} L^{b} \stackrel{\mathrm{pr}_{1}}{\longrightarrow} t_{\mathcal{A}}^{*} \longrightarrow 0
$$

is an exact sequence of $O$ - $R$-modules. (Here $\mathrm{pr}_{1}$ is induced by the natural projection $I_{A(G)^{b}} \longrightarrow t_{\mathcal{A}}^{*}$.) Note that with respect to the identification $L^{b}=L \oplus L^{(q)}, j_{L}: l_{2} \mapsto\left(0, l_{2}\right)$ and $\operatorname{pr}_{1}:\left(l_{1}, l_{2}\right) \mapsto l_{1}$ for any $l_{1} \in L$ and $l_{2} \in L^{(q)}$.

\subsection{The morphism $V_{\pi}(\mathcal{G})$}

With the notation from $\S 3.1$ consider the map $\psi_{\pi}:=[\pi]^{b}-\pi \operatorname{id}_{L^{b}}: L^{b} \longrightarrow L^{b}$. As the action of $\pi$ on $\mathcal{G}$ is strict, $\psi_{\pi}$ induces the zero morphisms on $N_{\mathcal{A}}$ and $t_{\mathcal{A}}^{*}$. From the exact sequences (6) and (7) it follows then that there is a unique $R$-linear morphism $V_{\pi}=V_{\pi}(\mathcal{G}): L \longrightarrow L^{(q)}$ such that $\psi_{\pi}=i_{L} \circ V_{\pi} \circ j_{L}$.

LEMMA. We have the following:

(a) $V_{\pi} \circ F_{q}=[\pi]-\pi \mathrm{id}_{L}$;

(b) $F_{q} \circ V_{\pi}=[\pi]^{(q)}-\pi \operatorname{id}_{L^{(q)}}$.

Proof. (a) The relation $j_{L} \circ i_{L}=F_{q}$ implies that

$$
i_{L} \circ\left([\pi]-\pi \operatorname{id}_{L}\right)=\left([\pi]^{b}-\pi \operatorname{id}_{L^{b}}\right) \circ i_{L}=i_{L} \circ V_{\pi} \circ j_{L} \circ i_{L}=i_{L} \circ\left(V_{\pi} \circ F_{q}\right) .
$$

Since $i_{L}$ is an epimorphism, we can cancel it from this equality to give the result. 


\section{Abrashikin}

(b) Take $l \in L, \hat{l} \in L^{b}$ such that $i_{L}(\hat{l})=l$ and compute in $A(G)^{b}$ :

$$
j_{L}\left(V_{\pi}\left(l^{q}\right)\right)=\left([\pi]^{b}-\pi \operatorname{id}_{L^{b}}\right)\left(\hat{l}^{q}\right)=\left([\pi]^{b}(\hat{l})\right)^{q}-\pi \hat{l}^{q}=j_{L}([\pi](l) \otimes 1-l \otimes \pi) .
$$

Since $j_{L}$ is a monomorphism we can cancel both sides by $j_{L}$ and obtain for any $l \in L$, that

$$
\left(F_{q} \circ V_{\pi}\right)(l \otimes 1)=[\pi]^{(q)}(l \otimes 1)-\pi \mathrm{id}_{L^{(q)}}(l \otimes 1) .
$$

The lemma is proved.

Remark. (a) For any $\mathcal{G} \in \operatorname{DGr}(O)_{R}, F_{q}$ induces the morphism $F_{\mathcal{G}} \in \operatorname{Hom}_{\operatorname{DGr}(O)_{R}}\left(\mathcal{G}, \mathcal{G}^{(q)}\right)$; this follows from the identity $F_{q} \circ[\pi]=[\pi]^{(q)} \circ F_{q}$.

(b) If $\pi \cdot 1_{R}=0$, then $V_{\pi}$ induces the morphism $V_{\mathcal{G}} \in \operatorname{Hom}_{\mathrm{DGr}(O)_{R}}\left(\mathcal{G}^{(q)}, \mathcal{G}\right)$ and we have $F_{\mathcal{G}} \circ V_{\mathcal{G}}=$ $\pi \operatorname{id}_{\mathcal{G}}$ and $V_{\mathcal{G}} \circ F_{\mathcal{G}}=\pi \operatorname{id}_{\mathcal{G}^{(q)}}$.

\subsection{The functor $\mathrm{L}_{q, \pi}: \operatorname{DGr}(O)_{R} \longrightarrow \operatorname{Mod}(O)_{R}$}

The category $\operatorname{Mod}(O)_{R}$ consists of triples $\left(L, F_{q}, V_{\pi}\right)$, where:

- $\left(L, F_{q}\right) \in \operatorname{Mod}\left(\mathbb{F}_{q}\right)_{R}$, i.e. $L$ is a locally free $R$-module of finite $R$-rank with an $R$-linear morphism $F_{q}: L^{(q)} \longrightarrow L$;

- $V_{\pi}: L \longrightarrow L^{(q)}$ is an $R$-linear morphism such that $\left(V_{\pi} \circ F_{q}+\pi \mathrm{id}_{L}\right)^{(q)}=F_{q} \circ V_{\pi}+\pi \mathrm{id}_{L^{(q)}}$;

- morphisms $f:\left(L, F_{q}, V_{\pi}\right) \longrightarrow\left(L_{1}, F_{q}, V_{\pi}\right)$ in $\operatorname{Mod}(O)_{R}$ are given by $R$-linear morphisms $f$ : $L \longrightarrow L_{1}$ such that $F_{q} \circ f=f^{(q)} \circ F_{q}$ and $V_{\pi} \circ f^{(q)}=f \circ V_{\pi}$.

Define the contravariant functor $\mathrm{L}_{q, \pi}$ from $\operatorname{DGr}(O)_{R}$ to $\operatorname{Mod}(O)_{R}$ by setting (where $\mathrm{L}_{q}$ is the functor from $\S 2.3)$ :

- $\mathrm{L}_{q, \pi}(\mathcal{G})=\left(L(\mathcal{G}), F_{q}, V_{\pi}(\mathcal{G})\right)$, where $\left(L(\mathcal{G}), F_{q}\right)=\mathrm{L}_{q}(\mathcal{G})$;

- if $\mathcal{H}=\left(H, H^{b}, i_{\mathcal{H}}\right) \in \operatorname{DGr}(O)_{R}$ and $f \in \operatorname{Hom}_{\operatorname{DGr}(O)_{R}}(\mathcal{G}, \mathcal{H})$, then $\mathrm{L}_{q, \pi}(f)=\mathrm{L}_{q}(f): L(\mathcal{H}) \longrightarrow$ $L(\mathcal{G})$ is an $R$-linear map induced by the corresponding map of $R$-algebras $A(H) \longrightarrow A(G)$.

Theorem 3. The functor $\mathrm{L}_{q, \pi}$ induces an antiequivalence of categories $\mathrm{L}_{q, \pi}^{*}: \operatorname{DGr}^{*}(O)_{R} \longrightarrow$ $\operatorname{Mod}(O)_{R}$.

Remark. The case of a perfect field $R$ of characteristic $p$ was considered in [Gib04].

Proof. Clearly, $\mathrm{L}_{q, \pi}$ is additive and faithful. We construct the inverse functor $\mathrm{D}_{q, \pi}: \operatorname{Mod}(O)_{R} \longrightarrow$ $\operatorname{DGr}^{*}(O)_{R}$ in the following way.

Let $\mathcal{L}=\left(L, F_{q}, V_{\pi}\right) \in \operatorname{Mod}(O)_{R}$. Take $\mathcal{G}=\mathrm{D}_{q}(\mathcal{L})=\operatorname{Spec}\left(A(G), A(G)^{b}, i_{\mathcal{A}}\right) \in \operatorname{DGr}\left(\mathbb{F}_{q}\right)_{R}$, where $\mathrm{D}_{q}$ is the functor from $\S 2.3 .1$, and provide it with a functorial strict action of $\pi$ as follows.

Define

$$
L^{q}:=\left\{\sum_{i} r_{i} l_{i}^{q} \mid l_{i} \in L, r_{i} \in R\right\} \subset \operatorname{Sym}_{R}(L) .
$$

Then the $R$-module $L^{q}$ can be identified with $L^{(q)}$ by the map

$$
\sum_{i} l_{i} \otimes r_{i} \mapsto\left(\sum_{i} l_{i} \otimes r_{i}\right)^{(q)}:=\sum_{i} r_{i} l_{i}^{q} .
$$

Consider the map of $R$-algebras $\phi_{\pi}: \operatorname{Sym}_{R}(L) \longrightarrow \operatorname{Sym}_{R}(L)$ such that $\phi_{\pi}: l \mapsto \pi l+V_{\pi}(l)^{(q)}$ for any $l \in L$. Then

$$
\begin{aligned}
\phi_{\pi}\left(l^{q}-F_{q}(l \otimes 1)\right) & =\phi_{\pi}(l)^{q}-\pi F_{q}(l \otimes 1)-\left(\left(F_{q} \circ V_{\pi}\right)(l \otimes 1)\right)^{(q)} \\
& =\pi^{q} l^{q}+\left(V_{\pi}(l)^{(q)}\right)^{q}-\pi F_{q}(l \otimes 1)-\left(\left(F_{q} \circ V_{\pi}\right)(l \otimes 1)\right)^{(q)} .
\end{aligned}
$$




\section{FALTings's STRICT MODULES}

From the definition of objects of $\operatorname{Mod}(O)_{R}$ we obtain

$$
\left(F_{q} \circ V_{\pi}\right)(l \otimes 1)^{(q)}=-\pi l^{q}+\pi^{q} l^{q}+\left(\left(V_{\pi} \circ F_{q}\right)(l)\right)^{q} .
$$

Substituting this in (8) we obtain for any $l \in L$,

$$
\phi_{\pi}\left(l^{q}-F_{q}(l \otimes 1)\right)=\pi\left(l^{q}-F_{q}(l \otimes 1)\right)+\left(V_{\pi}(l)^{(q)}-F_{q}\left(V_{\pi}(l)\right)\right)^{q} .
$$

Therefore, $\phi_{\pi}$ induces morphisms of $R$-algebras $[\pi]: A(G) \longrightarrow A(G)$ and $[\pi]^{b}: A(G)^{b} \longrightarrow A(G)^{b}$, which determine an action of $\pi$. This action is strict because $\left(V_{\pi}(l)^{(q)}-F_{q}\left(V_{\pi}(l)\right)\right)^{q} \in I \cdot I_{0}$.

So, we obtain an object of the category $\operatorname{DGr}(O)_{R}$ which will be denoted by $\mathrm{D}_{q, \pi}(\mathcal{L})$. Clearly, $\mathrm{L}_{q, \pi}\left(\mathrm{D}_{q, \pi}(\mathcal{L})\right)=\mathcal{L}$

Suppose that $\mathcal{L}_{1}=\left(L_{1}, F_{q}, V_{\pi}\right) \in \operatorname{Mod}(O)_{R}$ and $f \in \operatorname{Hom}_{\operatorname{Mod}(O)_{R}}\left(\mathcal{L}, \mathcal{L}_{1}\right)$. Then we have the $R$-linear morphism $f: L \longrightarrow L_{1}$ such that $f \circ V_{\pi}=V_{\pi} \circ f^{(q)}$ and $f^{(q)} \circ F_{q}=F_{q} \circ f$. Therefore, $f$ induces the morphism of $R$-algebras

$$
\psi_{f}: \operatorname{Sym}_{R}(L) \longrightarrow \operatorname{Sym}_{R}\left(L_{1}\right),
$$

which determines the morphism $\bar{\psi}_{f}$ in the category $\operatorname{Mod}\left(\mathbb{F}_{q}\right)_{R}$. It remains to prove that $\bar{\psi}_{f}$ commutes with the action of $\pi$ on $\mathrm{D}_{q, \pi}(\mathcal{L})$ and $\mathrm{D}_{q, \pi}\left(\mathcal{L}_{1}\right)$.

For any $l \in L$, let $V_{\pi}(l)=\sum l_{i} \otimes r_{i} \in L^{(q)}$. Then $V_{\pi}\left(\psi_{f}(l)\right)=V_{\pi}(f(l))=f^{(q)}\left(V_{\pi}(l)\right)=$ $\sum f\left(l_{i}\right) \otimes r_{i}=\sum \psi_{f}\left(l_{i}\right) \otimes r_{i}$. Therefore,

$$
\begin{aligned}
\psi_{f}\left(\phi_{\pi}(l)\right) & =\psi_{f}\left(\pi l+V_{\pi}(l)^{(q)}\right)=\psi_{f}\left(\pi l+\sum_{i} r_{i} l_{i}^{q}\right) \\
& =\pi \psi_{f}(l)+\sum r_{i} \psi_{f}(l)^{q}=\pi \psi_{f}(l)+V_{\pi}\left(\psi_{f}(l)\right)^{(q)}=\phi_{\pi}\left(\psi_{f}(l)\right) .
\end{aligned}
$$

So, $\bar{\psi}_{f} \in \operatorname{Hom}_{\mathrm{DGr}(O)_{R}}\left(\mathrm{D}_{q, \pi}\left(\mathcal{L}_{1}\right), \mathrm{D}_{q, \pi}(\mathcal{L})\right)$. It is easy to see that $\mathrm{L}_{q, \pi}\left(\bar{\psi}_{f}\right)=f$.

The theorem is proved.

Remark. The above theorem shows that the category of strict $O$-modules $\operatorname{DGr}^{*}(O)_{R}$ is equivalent to the category of finite $v$-modules introduced by Taguchi in [Tag95].

\section{The category of $\pi$-torsion strict modules}

Denote by $\operatorname{DGr}^{\prime}(O)_{R}$, respectively by $\operatorname{DGr}^{\prime *}(O)_{R}$, the full subcategory in $\operatorname{DGr}(O)_{R}$, respectively in $\operatorname{DGr}^{*}(O)_{R}$, consisting of ' $\pi$-torsion objects', i.e. of $\left(G, G^{b}\right) \in \operatorname{DGr}(O)_{R}$ such that $[\pi]_{G}: A(G) \longrightarrow$ $A(G)$ is nilpotent.

Clearly, the functor $\mathrm{L}_{q, \pi}$ induces an antiequivalence of $\mathrm{DGr}^{*}(O)_{R}$ and the full subcategory $\operatorname{Mod}^{\prime}(O)_{R}$ in $\operatorname{Mod}(O)_{R}$, which consists of $\left(L, F_{q}, V_{\pi}\right)$ such that $[\pi]_{L}=\pi \operatorname{id}_{L}+V_{\pi} \circ F_{q}$ is a nilpotent endomorphism of $L$.

4.1 Let $R^{*}$ be the multiplicative group of invertible elements of $R$.

Proposition. Suppose that $\pi \cdot 1_{R} \in R^{*}$ and $\mathcal{G}=\left(G, G^{b}\right) \in \operatorname{DGr}(O)_{R}$. Then $G$ is etale over $R$.

Proof. Let $\mathrm{L}_{q, \pi}(\mathcal{G})=\left(L, F_{q}, V_{\pi}\right)$. Then $[\pi]_{L}$ acts on $L / \operatorname{Im} F_{q}$ via the scalar multiplication by $\pi \cdot 1_{R} \in R^{*}$. On the other hand, this action should be nilpotent. Therefore, $L=\operatorname{Im} F_{q}$ and $G$ is etale over $R$.

Corollary. Suppose that $R$ is an integral domain, $K=\operatorname{Frac} R$ and $\Gamma_{K}=\operatorname{Gal}\left(K_{\mathrm{sep}} / K\right)$. If $\left(G, G^{b}\right) \in \operatorname{DGr}^{\prime}(O)_{R}$, then $G \otimes K$ is etale and $G\left(K_{\text {sep }}\right)$ is a finite $O\left[\Gamma_{K}\right]$-module of order $\operatorname{rk}_{R} A(G)$. 


\section{ABRASHKIN}

4.2 The following statement was conjectured in [Fal02] and is proved here following the referee's idea.

Theorem 4. Suppose that $\left(G, G^{b}\right) \in \operatorname{DGr}^{\prime}(O)_{R}$ and $\operatorname{rk}_{R} A(G)=q^{h}$, then $[\pi]_{G}^{h}=0$.

Note that for any $\mathcal{G}=\left(G, G^{b}\right) \in \operatorname{DGr}\left(\mathbb{F}_{q}\right)_{R}$ with $\mathrm{L}_{q}(\mathcal{G})=\left(L, F_{q}\right)$, we have $\operatorname{rk}_{R} A(G)=q^{h}$, where $h=\operatorname{rk}_{R} L$.

Via the antiequivalence $\mathrm{L}_{q, \pi}$, Theorem 4 can be obtained from the following proposition.

Proposition. Suppose that $R$ is a local ring, $\mathcal{L}=\left(L, F_{q}, V_{\pi}\right) \in \operatorname{Mod}^{\prime}(O)_{R}$ and $h=\operatorname{rk}_{R} L$. Then $[\pi]_{L}^{h}=0$.

Proof. Let $e_{1}, \ldots, e_{h}$ be an $R$-basis of $L$. Let $C=\left(c_{i j}\right) \in M_{h}(R)$ and $D=\left(d_{i j}\right) \in M_{h}(R)$ be such that for all $1 \leqslant j \leqslant h$,

$$
V_{\pi}\left(e_{j}\right)=\sum_{i} e_{i} \otimes c_{i j}, \quad F_{q}\left(e_{j} \otimes 1\right)=\sum_{i} e_{i} d_{i j} .
$$

Here and below we use the matrix notation $\bar{e}=\left(e_{1}, \ldots, e_{h}\right), V_{\pi}(\bar{e})=\bar{e} \otimes C$ and $F_{q}(\bar{e})=\bar{e} D$.

Then the $R$-linear morphism $V_{\pi} \circ F_{q}$ is given by the matrix $D C$ in the basis $e_{1}, \ldots, e_{n}$ and $F_{q} \circ V_{\pi}$, and by the matrix $C D$ in the basis $e_{1} \otimes 1, \ldots, e_{h} \otimes 1$. From the definition of objects of the category $\operatorname{Mod}(O)_{R}$ it follows that

$$
D^{(q)} C^{(q)}+\pi^{q} E=C D+\pi E,
$$

where $E$ is the unit matrix of order $h$ and $C^{(q)}=\left(c_{i j}^{q}\right), D^{(q)}=\left(d_{i j}^{q}\right)$. Note also that if $\Pi \in M_{h}(R)$ is such that $[\pi]_{L}(\bar{e})=\bar{e} \Pi$, then $D C+\pi E=\Pi$ and $C D+\pi E=\Pi^{(q)}$.

Let $\lambda$ be an indeterminate.

Lemma. We have $\operatorname{det}(C D-\lambda E)=\operatorname{det}(D C-\lambda E)$.

Proof. We can assume that all coefficients in $C$ and $D$ are independent variables over $\mathbb{Q}$. Then our lemma holds over a Zariski closed subset $\mathcal{V}$ in $\mathbb{A}_{\mathbb{Q}}^{2 h^{2}}$, which must contain the Zariski open subset $\operatorname{det} C \neq 0$ (where $C D$ and $D C$ become conjugate). Therefore, $\mathcal{V}=\mathbb{A}_{\mathbb{Q}}^{2 h^{2}}$. The lemma is proved.

Corollary. Let $\Phi(\lambda)$ be the characteristic polynomial for $[\pi]_{L}$. Then $\Phi(\lambda) \in \mathbb{F}_{q}[\lambda] \subset R[\lambda]$.

Proof. It will be sufficient to prove that $\Phi(\lambda)=\Phi^{(q)}(\lambda)$, where all coefficients of $\Phi^{(q)}$ are $q$ th powers of the corresponding coefficients of $\Phi$

$$
\begin{aligned}
\Phi(\lambda) & =\operatorname{det}(\Pi-\lambda E)=\operatorname{det}(D C+(\pi-\lambda) E) \\
& =\operatorname{det}(C D+(\pi-\lambda) E)=\operatorname{det}\left(\Pi^{(q)}-\lambda E\right)=\Phi(\lambda)^{(q)} .
\end{aligned}
$$

We now complete the proof of our proposition. Let $k$ be the residue field of $R$. Then $[\pi]_{L} \otimes k$ is a nilpotent endomorphism of $L \otimes k$ with the same characteristic polynomial $\Phi(\lambda) \in \mathbb{F}_{q}[\lambda] \subset k[\lambda]$. Therefore, $\Phi(\lambda)=\lambda^{h}$ and by the Cayley-Hamilton theorem $[\pi]_{L}^{h}=0$.

The proposition is proved.

The above method also gives the following property.

Corollary. Suppose that $\operatorname{Spec} R$ is connected, $x \in \operatorname{Spec} R$ with the residue field $k(x)$ and $\mathcal{G} \in$ $\operatorname{DGr}(O)_{R}$. Then $\mathcal{G} \in \operatorname{DGr}^{\prime}(O)_{R}$ if (and only if) $\mathcal{G} \otimes k(x) \in \operatorname{DGr}^{\prime}(O)_{k(x)}$. 


\section{FALTINGS'S STRICT MODULES}

\subsection{Embedding into a $\pi$-divisible group}

By $\S 1$ the objects $\mathcal{G}$ of the category $\operatorname{DGr}(O)_{R}$ can be treated as conventional group schemes with an endomorphism $[\pi]_{\mathcal{G}}$, which satisfies an extra condition of strictness. Therefore, we can consider short exact sequences in $\operatorname{DGr}(O)_{R}$ and define the concept of a $\pi$-divisible group following the original definition of Tate, cf. [Tat67]. Then a $\pi$-divisible group appears as an inductive system $\left\{\mathcal{G}_{n} \mid n \in \mathbb{N}\right\}$ of objects $\mathcal{G}_{n}=\left(G_{n}, G_{n}^{b}\right) \in \operatorname{DGr}(O)_{R}$ such that the corresponding inductive system $\left\{G_{n} \mid n \in \mathbb{N}\right\}$ is a $\pi$-divisible group in the category of finite flat $O$-module schemes over $R$. Note that for any $n \in \mathbb{N}$, $[\pi]_{\mathcal{G}_{n}}^{n}=0$ in $\operatorname{DGr}^{*}(O)_{R}$ and, therefore, $\mathcal{G}_{n} \in \operatorname{DGr}^{\prime}(O)_{R}$ for all $n \in \mathbb{N}$.

Theorem 5. Any $\mathcal{G} \in \operatorname{DGr}^{\prime}(O)_{R}$ admits locally on $R$ an embedding into a $\pi$-divisible group in the category $\operatorname{DGr}(O)_{R}$.

Proof. Suppose that $\mathrm{L}_{q, \pi}(\mathcal{G})=\left(L, F_{q}, V_{\pi}\right)$. Then we can assume that $L$ has an $R$-basis $\bar{e}=$ $\left(e_{1}, \ldots, e_{h}\right)$. Therefore, $V_{\pi}$ and $F_{q}$ can be given via the matrices $C, D \in M_{h}(R)$ such that with notation as in $\S 3.4, V_{\pi}(\bar{e})=\bar{e} \otimes C$ and $F_{q}(\bar{e} \otimes 1)=\bar{e} D$. Recall that $D C+\pi E=\Pi$ and $C D+\pi E=\Pi^{(q)}$, where $[\pi]_{L}(\bar{e})=\bar{e} \Pi$ and $\Pi^{(q)}=\sigma_{q} \Pi$.

For $N \in \mathbb{N}$, consider the inductive system of $R$-modules $\widetilde{L}_{N}=\bigoplus_{1 \leqslant n \leqslant 2 N} L_{n}$, where each $L_{n}$ is just a copy of the $R$-module $L$. Use the notation $\bar{e}_{n}$ for the basis $\bar{e}$ of $L_{n}$. Define the structural morphisms $\widetilde{V}_{\pi}: \widetilde{L}_{N} \longrightarrow \widetilde{L}_{N}^{(q)}$ and $\widetilde{F}_{q}: \widetilde{L}_{N}^{(q)} \longrightarrow \widetilde{L}_{N}$ by setting for $1 \leqslant n \leqslant N$,

$$
\begin{gathered}
\widetilde{V}_{\pi}\left(\bar{e}_{2 n}\right)=\bar{e}_{2 n} \otimes C+\bar{e}_{2 n-1} \otimes 1, \quad \widetilde{V}_{\pi}\left(\bar{e}_{2 n-1}\right)=-\bar{e}_{2 n-1} \otimes D-\bar{e}_{2 n} \otimes \Pi^{(q)}+\delta_{n 1}^{*} \bar{e}_{2 n-2} \otimes 1 \\
\widetilde{F}_{q}\left(\bar{e}_{2 n} \otimes 1\right)=\bar{e}_{2 n} D+\bar{e}_{2 n-1}, \quad \widetilde{F}_{q}\left(\bar{e}_{2 n-1} \otimes 1\right)=-\bar{e}_{2 n-1} C-\bar{e}_{2 n} \Pi+\delta_{n 1}^{*} \bar{e}_{2 n-2},
\end{gathered}
$$

where $\delta^{*}$ is the opposite Kronecker symbol, i.e. $\delta_{n 1}^{*}=0$ if $n=1$ and $\delta_{n 1}^{*}=1$, otherwise. Then a straightforward computation shows that for $1 \leqslant n \leqslant 2 N$,

$$
\begin{gathered}
\left(\widetilde{V}_{\pi} \circ \widetilde{F}_{q}+\pi \operatorname{id}_{\widetilde{L}_{N}}\right)\left(\bar{e}_{n}\right)=\delta_{n 1}^{*} \delta_{n 2}^{*} e_{n-2} \\
\left(\widetilde{F}_{q} \circ \widetilde{V}_{\pi}+\pi \operatorname{id}_{\widetilde{L}_{N}^{(q)}}\right)\left(\bar{e}_{n} \otimes 1\right)=\delta_{n 1}^{*} \delta_{n 2}^{*} e_{n-2} \otimes 1 .
\end{gathered}
$$

Therefore, each $\widetilde{L}_{N}$ is an object of the category $\operatorname{Mod}(O)_{R}$ and for all $1 \leqslant n \leqslant 2 N$, one has $[\pi]_{\widetilde{L}_{N}}\left(\bar{e}_{n}\right)=\delta_{n 1}^{*} \delta_{n 2}^{*} \bar{e}_{n-2}$. This means that $\left\{\widetilde{L}_{N} \mid N \geqslant 1\right\}$ is a $\pi$-divisible group in the category $\operatorname{Mod}(O)_{R}$. We have also an epimorphic map from $\left(\widetilde{L}_{h}, \widetilde{F}_{q}, \widetilde{V}_{\pi}\right)$ to $\left(L, F_{q}, V_{\pi}\right)$ given by the correspondences $\bar{e}_{2 n} \mapsto[\pi]_{L}^{h-n}(\bar{e})$ and $\bar{e}_{2 n-1} \mapsto \overline{0}$ if $1 \leqslant n \leqslant h$.

Finally, applying the antiequivalence $\mathrm{L}_{q, \pi}$ we obtain the statement of our theorem.

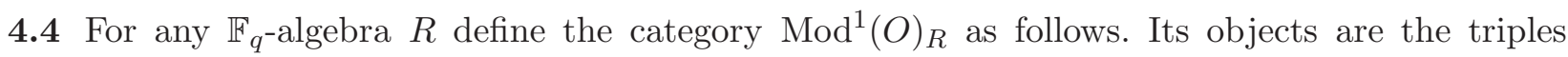
$\left(L, F_{q}, \Pi\right)$ such that:

- $\left(L, F_{q}\right) \in \operatorname{Mod}\left(\mathbb{F}_{q}\right)_{R}$ and $F_{q}$ is injective;

- $\Pi \in \operatorname{End}_{R}(L)$ is nilpotent, $\Pi^{(q)} \circ F_{q}=F_{q} \circ \Pi$ and for any $l \in L, \Pi(l) \equiv \pi l \bmod \left(\operatorname{Im} F_{q}\right)$.

The morphisms $f:\left(L, \mathbb{F}_{q}\right) \longrightarrow\left(L_{1}, F_{q}\right)$ in $\operatorname{Mod}^{1}(O)_{R}$ arise from the morphisms of the category $\operatorname{Mod}\left(\mathbb{F}_{q}\right)_{R}$ that commute with $\Pi$.

Consider the functor $M_{\pi}^{1}: \operatorname{Mod}^{\prime}(O)_{R} \longrightarrow \operatorname{Mod}^{1}(O)_{R}$ given by the correspondence $\left(L, F_{q}, V_{\pi}\right) \mapsto$ $\left(L, F_{q},[\pi]_{L}\right)$. The following theorem easily follows from the proposition of $\S 4.1$.

Theorem 6. If $R$ is an integral domain then the functor $\mathrm{L}_{q}^{1}=\mathrm{L}_{q, \pi} \circ M_{\pi}^{1}$ gives an antiequivalence of the categories $\operatorname{DGr}^{* \prime}(O)_{R}$ and $\operatorname{Mod}^{1}(O)_{R}$. 


\section{ABRASHKIN}

\subsection{Relation to the mixed characteristic case}

In this section $R$ is the valuation ring in a complete discrete valuation field $K$ of characteristic $p$. We denote by $k$ the residue field in $K$ and by $\eta$ one of its uniformisers. We assume also that the $O$-algebra structure on $R$ is given via the embedding $O \subset R$ such that $\pi \notin R^{*}$. Then $\hat{O}=\mathbb{F}_{q}[[\pi]]$ is the valuation ring of a complete discrete valuation subfield $E$ in $K$. We denote below by $e(K / E)$ the ramification index of $K$ over $E$.

With the above notation introduce the full subcategory $\operatorname{DGr}_{1}(O)_{R}$ in $\operatorname{DGr}^{*}(O)_{R}$ consisting of objects killed by $[\pi]$. Via the functor $\mathrm{L}_{q}^{1}$ this category is antiequivalent to the full subcategory $\operatorname{Mod}_{1}^{1}(O)_{R}$ in $\operatorname{Mod}^{1}(O)_{R}$ consisting of the objects $\left(L, F_{q}, 0\right)$.

4.5.1 Suppose that $e(K / E)=1$. Introduce the category $\mathrm{SH}_{1}\left(\mathbb{F}_{q}\right)_{R}$ with objects $\left(M_{0}, M_{1}\right.$, $\left.\varphi_{0}, \varphi_{1}\right)$, where $M_{0}$ is an $R$-module of finite length such that $\pi M_{0}=0, \varphi_{0}: M_{0}^{(q)} \longrightarrow M_{0}$ is an $R$-linear morphism, $M_{1}=\operatorname{Ker} \varphi_{0}, \varphi_{1}: M_{1}^{(q)} \longrightarrow M_{0}$ is an $R$-linear morphism and $\operatorname{Im} \varphi_{0}+\operatorname{Im} \varphi_{1}=M$. This is an analogue of Fontaine's category of 'filtered modules of length 1 killed by $p$ '.

Consider the functor $\mathrm{SH}: \operatorname{Mod}_{1}^{1}(O)_{R} \longrightarrow \mathrm{SH}_{1}\left(\mathbb{F}_{q}\right)_{R}$ defined by the correspondence $\left(L, F_{q}, 0\right) \mapsto$ $\left(M_{0}, M_{1}, \varphi_{0}, \varphi_{1}\right)$, where $M_{0}=L \bmod \pi L, \varphi_{0}=F_{q} \bmod \pi L$ and $\varphi_{1}$ is induced by $\frac{1}{\pi} F_{q}$. The functor $\mathrm{SH}$ is an equivalence of categories if $q>2$ and is 'very close' to such an equivalence if $q=2$. This shows that strict $O$-modules have a similar description as conventional group schemes in the case $e(K / E)=1$.

4.5.2 Suppose that $e(K / E) \leqslant q-1$. Define the category $\operatorname{SH}_{1}(O)_{R}$ of the collections $\left(M, M_{0}\right.$, $\left.M_{1}, \varphi_{0}, \varphi_{1}\right)$, where $M$ is an $R$-module of finite length killed by $\pi, M_{0}=\{m \in M \mid \eta m=0\}$, $\varphi_{0}: M_{0}^{(q)} \longrightarrow M$ is an $R$-linear map, $M_{1}=\operatorname{Ker} \varphi_{0}, \varphi_{1}: M_{1}^{(q)} \longrightarrow M$ is an $R$-linear map, and $\operatorname{Im} \varphi_{0}+\operatorname{Im} \varphi_{1}=M$. This category is an analogue of the category $\mathrm{SH}_{O}$ from[Abr90].

Consider the functor $\mathrm{SH}: \operatorname{Mod}_{1}^{1}(O)_{R} \longrightarrow \mathrm{SH}_{1}(O)_{R}$ defined by the correspondence $\left(L, F_{q}, 0\right) \mapsto$ $\left(M, M_{0}, M_{1}, \varphi_{0}, \varphi_{1}\right)$, where:

- $M$ is the image of $\frac{\eta}{\pi} F_{q}\left(L^{(q)}\right)+\frac{1}{\pi} F_{q}\left(L_{1}^{(q)}\right)$ in $\frac{\eta}{\pi} L \bmod (\eta L)$ with $L_{1}^{(q)}=\left\{l \in L^{(q)} \mid F_{q}(l) \in \eta L\right\}$;

- $M_{0}=L \bmod \eta L$ and $\varphi_{0}: M_{0}^{(q)} \longrightarrow M$ is induced by $\frac{\eta}{\pi} F_{q}$;

- $M_{1}=L_{1}^{(q)} \bmod \eta L$ and $\varphi_{1}: M_{1}^{(q)} \longrightarrow M$ is induced by $\frac{1}{\pi} F_{q}$.

If $e<q-1$ then $\mathrm{SH}$ is an equivalence of categories and, if $e=q-1$, then $\mathrm{SH}$ is 'very close' to such an equivalence.

Note that when working in the equal characteristic case, the category $\mathrm{SH}_{1}(O)_{R}$ can be replaced by a simpler category $\mathrm{SH}_{1}^{\prime}(O)_{R}$ consisting of triples $\left(M, M_{0}, \varphi\right)$, where $\bar{M}$ is an $R$-module of finite length killed by $\pi, M_{0}=\{m \in M \mid \eta m=0\}$ and $\varphi: M_{0}^{(q)} \longrightarrow M$ is an $R$-linear morphism such that $\operatorname{Im} \varphi=M$. The functor $\operatorname{SH}^{\prime}: \operatorname{Mod}_{1}^{1}(O)_{R} \longrightarrow \mathrm{SH}_{1}^{\prime}(O)_{R}$ is defined by the correspondence $\left(L, F_{q}, 0\right) \mapsto\left(M, M_{0}, \varphi\right)$, where $M=\frac{1}{\pi} L^{(q)} \bmod \eta L, M_{0}=L \bmod \eta L$ and $\varphi$ is induced by $\frac{1}{\pi} F_{q}$ (this map is additive because of the equal characteristic assumption).

4.5.3 Suppose that $e(K / E)$ is arbitrary. Introduce the category $\operatorname{BR}_{1}(O)_{R}$. Its objects are the triples $\left(M, M_{1}, \varphi\right)$, where $M$ is an $R$-module of finite length such that $\pi M=0, M_{1}$ is an $R_{1}$-submodule in $M \otimes_{R} R_{1}$, where $R_{1}=R\left[\eta_{1}\right]$ with $\eta_{1}^{q}=\eta$, and $\varphi: M_{1}^{(q)} \longrightarrow M$ is an $R$-linear map such that $\varphi\left(M_{1}\right)=M \otimes_{R} R_{1}$. This category is an equicharacteristic version of Breuil's category $\operatorname{Mod}_{/ S_{1}}$ that appeared in his classification of period $p$ group schemes in the mixed characteristic case, cf. [Bre00].

Again there is a natural functor from $\operatorname{Mod}_{1}^{1}(O)_{R}$ to $\operatorname{BR}_{1}(O)_{R}$ defined by the correspondence $\left(L, F_{q}, 0\right) \mapsto\left(M, M_{1}, \varphi\right)$, where $M=L \bmod \pi L$ and $\varphi$ is induced by $\frac{1}{\pi} F_{q}$. 


\section{FALTINGS'S STRICT MODULES}

4.5.4 Again $e\left(K / K_{0}\right)$ is arbitrary. Introduce an equal characteristic analogue of the concept of ' $p$-etale $\varphi$-module of $q$-height $r$ over $S$ ' from [Fon90].

Let $r \in \mathbb{N}$. Introduce the category $\operatorname{Mod}^{r}(O)_{R}$ by generalizing the definition of $\operatorname{Mod}^{1}(O)_{R}$ from $\S$ 4.4. Its objects are $\left(L, F_{q}, \Pi\right)$, where:

- $\left(L, F_{q}\right) \in \operatorname{Mod}\left(\mathbb{F}_{q}\right)_{R}$ and $F_{q}$ is injective;

- $\Pi \in \operatorname{End}_{R} L$ is nilpotent and satisfies the relation $F_{q} \circ \Pi=\Pi^{(q)} \circ F_{q}$;

- for any $l \in L,\left(\Pi-\pi \operatorname{id}_{L}\right)^{r}(l) \in \operatorname{Im} F_{q}$.

The morphisms in this category are morphisms of the category $\operatorname{Mod}(O)_{R}$, which commute with $\Pi$.

Let $\mathrm{MG}(\mathrm{O})_{\mathrm{K}}$ be the category of finite continuous $O\left[\Gamma_{K}\right]$-modules. Consider the functor MG : $\operatorname{Mod}^{\mathrm{r}}(\mathrm{O})_{\mathrm{R}} \longrightarrow \mathrm{MG}(\mathrm{O})_{\mathrm{K}}$ defined by the correspondence $\left(L, F_{q}, \Pi\right) \mapsto G\left(K_{\text {sep }}\right)$, where $\mathrm{D}_{q}\left(L, F_{q}\right)=$ $\left(G, G^{b}\right) \in \operatorname{DGr}\left(\mathbb{F}_{q}\right)_{R}$, cf. $\S 2.3 .1$, and the action of $O=\mathbb{F}_{q}[\pi]$ on $G\left(K_{\text {sep }}\right)$ comes from the $\mathbb{F}_{q}$-action on $G$ and the action of $\pi$ via the morphism $\mathrm{D}_{q}(\Pi)$.

For any $r \in \mathbb{N}$, let $\mathrm{MG}^{\mathrm{r}}(\mathrm{O})_{\mathrm{K}}$ be the full subcategory in $\mathrm{MG}(\mathrm{O})_{\mathrm{K}}$ consisting of the $O\left[\Gamma_{K}\right]$-modules $\operatorname{MG}(\mathcal{L})$, where $\mathcal{L} \in \operatorname{Mod}^{r}(O)_{R}$. The study of properties of its objects appears as equicharacteristic analogue of the problem of study of finite subquotients of crystalline representations with HodgeTate weights from $[0, r]$. If $r=1$, then objects of $\mathrm{MG}^{1}(\mathrm{O})_{\mathrm{K}}$ come from the Galois modules of generic fibres of strict $O$-modules over $R$.

\subsection{Several remarks about duality in $\operatorname{DGr}^{*}(O)_{R}$}

Suppose that $\mathcal{L}=\left(L, F_{q}, V_{\pi}\right) \in \operatorname{Mod}(O)_{R}$. Let $L^{D}=\operatorname{Hom}_{R \text {-mod }}(L, R)$. Then $L^{D}$ is a locally free $R$-module and $L^{D(q)}=\operatorname{Hom}_{R \text {-mod }}\left(L^{(q)}, R\right)$. Therefore, we have $R$-linear maps $F_{q}: L^{D(q)} \longrightarrow L^{D}$ and $V_{\pi}: L^{D} \longrightarrow L^{D(q)}$ such that for any $\tilde{l} \in L^{D}$ and $l \in L, F_{q}(\tilde{l} \otimes 1)(l)=(\tilde{l} \otimes 1)\left(V_{\pi} l\right)$ and $V_{\pi}(\tilde{l})(l \otimes 1)=\tilde{l}\left(F_{q}(l \otimes 1)\right)$.

It can be easily verified that $\mathcal{L}^{D}:=\left(L^{D}, F_{q}, V_{\pi}\right) \in \operatorname{Mod}(O)_{R}$. The correspondence $\mathcal{L} \mapsto \mathcal{L}^{D}$ gives a perfect duality in $\operatorname{Mod}(O)_{R}$ that has all nice functorial properties. This duality was introduced and studied in [Tag95].

Consider the formal Lubin-Tate group $G_{L T}=\operatorname{Spf} R[[X]]$ such that $\operatorname{End}_{R}\left(G_{L T}\right)=\hat{O}$ and the endomorphism $[\pi]_{L T}$ of multiplication by $\pi \in O$ is given by $[\pi]_{L T}(X)=X^{q}+\pi X$. Then for $h \in \mathbb{N}, G_{L T, h}:=\operatorname{Ker}[\pi]_{L T}^{h}$ has a natural structure of an object $\mathcal{G}_{L T, h}$ of the category $\operatorname{DGr}(O)_{R}$. Let $\mathrm{L}_{q, \pi}\left(\mathcal{G}_{L T, h}\right)=\mathcal{L}_{L T, h}=\left(L_{h}, F_{q}, V_{\pi}\right)$. Then $\mathcal{L}_{L T, h} \in \operatorname{Mod}^{\prime}(O)_{R}$ and $L_{h}=\bigoplus_{1 \leqslant i \leqslant h} R m_{i}$, where for $1 \leqslant i \leqslant h, F_{q}\left(m_{i} \otimes 1\right)=m_{i-1}-\pi m_{i}$ and $V_{\pi}\left(m_{i}\right)=m_{i} \otimes 1$ with the agreement $m_{0}=0$.

Suppose that $\mathcal{L}=\left(L, F_{q}, V_{\pi}\right) \in \operatorname{Mod}^{\prime}(O)_{R}$ and $[\pi]_{L}^{h}=0$. Then $\mathcal{L}^{D}=\left(L^{D}, F_{q}, V_{\pi}\right) \in \operatorname{Mod}^{\prime}(O)_{R}$ and $[\pi]_{L^{D}}^{h}=0$. Consider the $R$-linear map

$$
c: L_{h} \longrightarrow L \otimes L^{D}=\operatorname{Hom}_{R}(L, L),
$$

which is uniquely determined by the requirements $c(m)=\mathrm{id}_{L}$ and $c \circ F_{q}=F_{q} \circ c^{(q)}$. Consider the natural embeddings $L_{h} \subset A\left(G_{L T, h}\right), L \subset A(G)$ and $L^{D} \subset A\left(G^{D}\right)$, where $\mathcal{G}=\left(G, G^{b}\right)$ and $\mathcal{G}^{D}=\left(G^{D}, G^{D b}\right)$ are such that $\mathrm{L}_{q, \pi}(\mathcal{G})=\mathcal{L}$ and $\mathrm{L}_{q, \pi}\left(\mathcal{G}^{D}\right)=\mathcal{L}^{D}$. Together with the above map $c$ these embeddings determine a morphism of $R$-schemes $G \times G^{D} \longrightarrow G_{L T, h}$, which induces a nondegenerate bilinear pairing of strict $O$-modules

$$
C: \mathcal{G} \times \mathcal{G}^{D} \longrightarrow \mathcal{G}_{L T, h}
$$

This is an analogue of the Cartier duality. Its direct construction is given in [Fal02]. In the context of finite $v$-modules, cf. the remark at the end of $\S 3.3$, the pairing $C$ appears in [Tag95].

Remark. The referee pointed out that the existence of the pairing $C$ implies a short proof of the difficult part of our Theorem 1 from $\S 2.1$. 


\section{ABRASHKIN}

\section{Properties of Galois modules from $\mathrm{MG}^{1}(O)_{K}$}

As in $\S 4.5, E \subset K$ are complete discrete valuation fields of characteristic $p$ with uniformisers $\pi$ and, respectively, $\eta$. The valuation ring of $E$ is $\hat{O}=\mathbb{F}_{q}[[\pi]]$ and the residue field $k$ of $K$ is perfect and the valuation ring of $K$ is $R$.

Suppose that $\mathcal{G}=\left(G, G^{b}\right) \in \operatorname{DGr}^{\prime}(O)_{R}, H=G\left(K_{\text {sep }}\right)$ is the $O\left[\Gamma_{K}\right]$-module of geometric points of $G$ and $e=e(K / E)$ - is the ramification index of $K$ over $E$. We also set $\Gamma_{K}=\operatorname{Gal}\left(K_{\mathrm{sep}} / K\right)$ and denote by $I_{K}$ the inertia subgroup of $\Gamma_{K}$.

\subsection{Characters of the semisimple envelope of $H$}

Suppose that $\bar{k}$ is an algebraic closure of $k$ and the character $\chi: I_{K} \longrightarrow \bar{k}^{*}$ appears with a nonzero multiplicity in the semisimple envelope of the $O\left[\Gamma_{K}\right]$-module $H$. An analogue of the Serre conjecture for $H$ can be stated as follows.

Theorem 7. For the above character $\chi$, there are $a, N \in \mathbb{N} \backslash p \mathbb{N}$ such that $\chi=\chi_{N}^{a}$, where $a=$ $a_{0}+a_{1} q+\cdots+a_{N-1} q^{N-1}$ with $0 \leqslant a_{i} \leqslant e$ and $\chi_{N}: I_{K} \longrightarrow \bar{k}^{*}$ is such that for any $\tau \in I_{K}$, $\chi_{N}(\tau)=\tau\left(\eta_{N}\right) / \eta_{N}$, where $\eta_{N} \in K_{\text {sep }}$ and $\eta_{N}^{q^{N}-1}=\eta$.

Proof. This can be deduced in the same way as it has been obtained in the case of usual group schemes in [Ray74]. First, we can assume that $k=\bar{k}$ and $e<q-1$. Then any simple object of the category $\operatorname{Mod}^{1}(O)_{R}$ appears in the form $\left(L, F_{q}, 0\right)$, where $L=\bigoplus_{0 \leqslant i<n} R m_{i}, F_{q} m_{0}=$ $\eta^{a_{0}} m_{1}, \ldots, F_{q} m_{N-1}=\eta^{a_{N-1}} m_{0}$ with $0 \leqslant a_{i} \leqslant e, 0 \leqslant i<N$.

Then the corresponding Galois module consists of $K_{\text {sep }}$-points of the $R$-algebra $R\left[T_{0}, \ldots, T_{N-1}\right]$, where $T_{0}^{q}=\eta^{a_{0}} T_{1}, \ldots, T_{N-1}^{q}=\eta^{a_{N-1}} T_{0}$. It can be naturally identified with the $\mathbb{F}_{q}\left[\Gamma_{K}\right]$-module $\left\{\alpha \eta_{N}^{a} \mid \alpha \in \mathbb{F}_{q^{N}}\right\}$, where $a=a_{0}+a_{1} q+\cdots+a_{N-1} q^{N-1}$. Clearly, $I_{K}$ acts on it via the conjugacy class of characters $\left\{\sigma^{i} \chi_{N}^{a} \mid 0 \leqslant i<N\right\}$.

Following Raynaud's method, cf. [Ray74], one can deduce from the above description of simple objects in $\operatorname{Mod}^{1}(O)_{R}$ that if $e<q-1$, then the functor $\left(G, G^{b}\right) \mapsto G\left(K_{\text {sep }}\right)$ is a fully faithful functor from $\operatorname{DGr}^{\prime}(O)_{R}$ to the category $\mathrm{MG}(\mathrm{O})_{\mathrm{K}}$ of finite $O\left[\Gamma_{K}\right]$-modules. The proof uses induction on the length of the Jordan-Hoelder series for $G\left(K_{\text {sep }}\right)$ and the description of simple objects in $\operatorname{Mod}^{1}(O)_{R}$ from the proof of the above Theorem 7 .

\subsection{Ramification estimates}

These estimates are given in Theorem 8 below and are very similar to the known estimates in the case of conventional group schemes, cf. [Fon85]. The proof is based on the knowledge of 'equations' of the strict module $G$ and is done below by the methods of [Abr98]. Note that the methods from [Fon93] can also be adjusted to obtain similar estimates, cf. also [Tag92]. Our method is simpler, but it works only in positive characteristic.

THEOREM 8. If $H$ is killed by $\left[\pi^{N}\right]$, then the ramification subgroups $\Gamma_{K}^{(v)}$ act trivially on $H$ for $v>e(N+1 /(q-1))-1$.

Proof. By Theorem 5 we can assume that there is a $\pi$-divisible group $\left\{\mathcal{G}_{n}\right\}_{n \geqslant 1}$ of a height $h$ in $\operatorname{DGr}(O)_{R}$ such that $\mathcal{G}_{N}=\mathcal{G}$.

5.2.1 Let $\mathrm{L}_{q}^{1}(\mathcal{G})=\left(L, F_{q}, \Pi\right)$, cf. $\S$ 4.4. Then $L$ is a free $R$-module of rank $h N$ and we can choose its $R$-basis in the form

$$
m_{1}, \ldots, m_{h}, \Pi m_{1}, \ldots, \Pi m_{h}, \ldots, \Pi^{N-1} m_{1}, \ldots, \Pi^{N-1} m_{h} .
$$




\section{FALTINGS'S STRICT MODULES}

For $1 \leqslant i \leqslant N$, introduce the vector-columns $\bar{m}^{(i)}=\left(\Pi^{N-i} m_{1}, \ldots, \Pi^{N-i} m_{h}\right)^{\mathrm{t}}$ and set $F_{q} \bar{m}^{(i)}=$ $\left(F_{q}\left(\Pi^{N-i} m_{1}^{\mathrm{t}}\right), \ldots, F_{q}\left(\Pi^{N-i} m_{h}^{\mathrm{t}}\right)\right)^{\mathrm{t}}$. Then, the condition $\operatorname{Im}\left(\Pi-\pi \mathrm{id}_{L}\right) \subset \operatorname{Im} F_{q}$ implies the existence of unique matrices $C_{1}, \ldots, C_{N} \in M_{h}(R)$ such that

$$
\begin{gathered}
C_{1} F_{q} \bar{m}^{(N)}+C_{2} F_{q} \bar{m}^{(N-1)}+\cdots+C_{N} F_{q} \bar{m}^{(1)}=\pi \bar{m}^{(N)}-\bar{m}^{(N-1)} \\
C_{1} F_{q} \bar{m}^{(N-1)}+\cdots+C_{N-1} F_{q} \bar{m}^{(1)}=\pi \bar{m}^{(N-1)}-\bar{m}^{(N-2)} \\
\vdots \\
C_{1} F_{q} \bar{m}^{(1)}=\pi \bar{m}^{(1)} .
\end{gathered}
$$

Note that $C_{1}$ divides $\pi E_{h}$, where $E_{h}$ is the unit matrix of order $h$.

Consider the vector columns $\bar{X}_{i}=\left(X_{i 1}, \ldots, X_{i h}\right)^{\text {t }}$ of independent variables $X_{i j}, 1 \leqslant i \leqslant N$, $1 \leqslant j \leqslant h$. Then the algebra $A(G) \otimes_{R} K$ appears as the quotient of $K\left[\bar{X}_{1}, \ldots, \bar{X}_{N}\right]$ by the ideal generated by the equations

$$
\sum_{1 \leqslant i \leqslant s} C_{i} \bar{X}_{s+1-i}^{q}=\pi \bar{X}_{s}-\bar{X}_{s-1}
$$

where $1 \leqslant s \leqslant N$ and by definition $\bar{X}_{0}=\overline{0}$.

Consider the points of $G\left(K_{\mathrm{sep}}\right)$ as solutions $\bar{a}=\left(\bar{a}_{1}, \ldots, \bar{a}_{N}\right)$ of the system (9).

LEMma. We have the following.

(a) If $\bar{a}=\left(\bar{a}_{1}, \ldots, \bar{a}_{N}\right) \in G\left(K_{\mathrm{sep}}\right)$, then $\bar{a}_{1}, \ldots, \bar{a}_{N}$ have coordinates in $O_{K_{\mathrm{sep}}}$.

(b) If $\bar{a}^{\prime}=\left(\bar{a}_{1}^{\prime}, \ldots, \bar{a}_{N}^{\prime}\right) \in G\left(K_{\mathrm{sep}}\right)$ and $\bar{a} \equiv \bar{a}^{\prime} \bmod \pi^{1 /(q-1)} m_{\text {sep }}$, where $m_{\text {sep }}$ is the maximal ideal of the valuation ring of $K_{\mathrm{sep}}$, then $\bar{a}=\bar{a}^{\prime}$.

Proof. Both statements follow easily by induction on $N$ from the above equations for points of $G$. Statement (b) requires only that $C_{1}, \ldots, C_{h} \in M_{h}(R)$, in statement (a) we need also that $C_{1}$ divides $\pi E_{h}$.

5.2.2 Suppose that $\alpha \in \mathbb{Q}>0$ has the zero $p$-adic valuation. Then $\alpha=m /\left(q^{M}-1\right)$ with suitable $m, M \in \mathbb{N},(m, p)=1$. Note that the presentation of $\alpha$ in the form of fraction $m /\left(q^{M}-1\right)$ is not unique and can always be chosen with an arbitrary large value of $M$. For any such $\alpha$ there is an extension $K_{\alpha}$ of $K$ of degree $q^{M}$ with the Herbrand function

$$
\varphi_{K_{\alpha} / K}(x)= \begin{cases}x, & \text { for } 0 \leqslant x \leqslant \alpha \\ \alpha+\frac{x-\alpha}{q^{M}}, & \text { for } x \geqslant \alpha .\end{cases}
$$

Note that $\varphi_{K_{\alpha} / K}$ has only one edge point $x=\alpha$.

Explicit construction of $K_{\alpha}$ can be found in [Abr98] and can be briefly described as follows. Let $\eta_{M} \in K_{\text {sep }}$ be such that $\eta_{M}^{q^{M}-1}=\eta$ and let $L_{\alpha}=K\left(\eta_{M}\right)(T)$, where

$$
T^{q^{M}}-T=\eta_{M}^{-m}
$$

Then $K_{\alpha}=K\left(\eta_{\alpha}\right)$, where $\eta_{\alpha}$ is a uniformising element in $K_{\alpha}$ such that $\eta_{\alpha}^{-m}=T^{q^{M}-1}$. From the above construction of $K_{\alpha}$, it follows that

$$
\eta_{\alpha}^{q^{M}}\left(1-\eta_{\alpha}^{m}\right)^{-1 / \alpha}=\eta,
$$

where $(-1 / \alpha)$ th power is taken via the binomial series. In particular,

$$
\eta \equiv \eta_{\alpha}^{q^{M}} \bmod \left(\eta \eta_{\alpha}^{m}\right)
$$




\section{Abrashikin}

Note that $K_{\alpha}$ is totally ramified over $K$ and, therefore, there is a field isomorphism $h_{\alpha}$ : $K \longrightarrow K_{\alpha}$ such that $h_{\alpha}(\eta)=\eta_{\alpha}$ and $\left.h_{\alpha}\right|_{k}=\mathrm{id}$. (Here $k$ is the residue field of $K$ and $K_{\alpha}$.)

Relation (10) now implies that for any $a \in \eta R, a=h_{\alpha}(a)^{q^{M}}+\tilde{a}$, with $\tilde{a} \in K_{\alpha}$ such that $v_{K}(\tilde{a}) \geqslant v_{K}(a)+\alpha\left(1-q^{-M}\right)\left(v_{K}\right.$ is the valuation in $K$ such that $\left.v_{K}(\eta)=1\right)$. Below we use the following consequence of this fact: if $a \in R$, then $v_{K}\left(a-h_{\alpha}(a)^{q^{M}}\right) \geqslant 1+\alpha\left(1-q^{-M}\right)$.

5.2.3 Denote by the same symbol an extension of $h_{\alpha}$ to an isomorphism of $K_{\text {sep onto }}$ $K_{\alpha, \text { sep }}=K_{\text {sep }}$. Clearly, $\bar{X}=\left(\bar{X}_{1}, \ldots, \bar{X}_{s}\right) \mapsto h_{\alpha}(\bar{X})=\left(h_{\alpha}\left(\bar{X}_{1}\right), \ldots, h_{\alpha}\left(\bar{X}_{s}\right)\right)$ is a one-to-one correspondence between solutions of the system (9) and solutions $\bar{Y}=\left(\bar{Y}_{1}, \ldots, \bar{Y}_{N}\right)$ of the similar system

$$
\sum_{1 \leqslant i \leqslant s} h_{\alpha}\left(C_{i}\right) \bar{Y}_{s+1-i}^{q}=h_{\alpha}(\pi) \bar{Y}_{s}-\bar{Y}_{s-1}
$$

where $1 \leqslant s \leqslant N$ and by definition $\bar{Y}_{0}=\overline{0}$.

Lemma. If $\alpha\left(1-1 / q^{M}\right)>e(N+1 /(q-1))-1$, then for any solution $\bar{X}^{(0)}$ of $(9)$ there is a unique solution $\bar{Y}^{(0)}$ of (11) such that $\bar{X}^{(0)} \equiv \bar{Y}^{(0) q^{M}} \bmod \pi^{1 /(q-1)} m_{\text {sep }}$.

Proof. The correspondence $\bar{Y} \mapsto \bar{Z}=\bar{Y}^{q^{M}}-\bar{X}^{(0)}$ establishes a one-to-one correspondence between solutions $\bar{Y}$ of (11) and solutions $\bar{Z}=\left(\bar{Z}_{1}, \ldots, \bar{Z}_{N}\right)$ of the system of equations

$$
\sum_{1 \leqslant i \leqslant s} C_{i} \bar{Z}_{s+1-i}^{q}=\pi \bar{Z}_{s}-\bar{Z}_{s-1}-\bar{F}_{s}
$$

where $1 \leqslant s \leqslant N, \bar{Z}_{0}=0$ and

$$
\bar{F}_{s}=\tilde{\pi} \bar{Y}_{s}^{q^{M}}-\sum_{1 \leqslant i \leqslant s} \tilde{C}_{i} \bar{Y}_{s+1-i}^{q^{M+1}} \in \pi^{N+1 /(q-1)} m_{\mathrm{sep}}
$$

because the $v_{K}$-valuations of $\tilde{\pi}=\pi-h_{\alpha}(\pi)^{q^{M}}$ and of $\tilde{C}_{i}=C_{i}-h_{\alpha}\left(C_{i}\right)^{\left(q^{M}\right)}$ are strictly bigger than $e(N+1 /(q-1))$.

Now induction on $s$ shows that the system (12) has a unique solution $\bar{Z}=\left(Z_{1}, \ldots, Z_{N}\right)$ with coordinates in $\pi^{1 /(q-1)} m_{\text {sep. }}$. (Note that these coordinates $Z_{1}, \ldots, Z_{N}$ are such that for $1 \leqslant s \leqslant N$, $\bar{Z}_{s} \equiv \overline{0} \bmod \pi^{N-s+1 /(q-1)} m_{\text {sep }}$.)

The lemma is proved.

With the above notation and assumptions we have the following corollary.

Corollary. Suppose that $L$, respectively $L_{\alpha}$, is obtained by joining to $K$, respectively $K_{\alpha}$, all coordinates of all solutions of the system of equations (9), respectively (11), in $K_{\mathrm{sep}}$. Then $L K_{\alpha}=L_{\alpha}$.

Proof. Suppose that $\tau \in \operatorname{Gal}\left(K_{\mathrm{sep}} / K_{\alpha}\right)$. Then $\tau \in \operatorname{Gal}\left(K_{\mathrm{sep}} / L K_{\alpha}\right)$ if and only if for any solution $\bar{X}^{(0)}$ of the system (9), we have (cf. the lemma in $\S 5.2 .1$ )

$$
\tau \bar{X}^{(0)} \equiv \bar{X}^{(0)} \bmod \pi^{1 /(q-1)} m_{\mathrm{sep}}
$$

By the above lemma this is equivalent to the congruences

$$
\tau \bar{Y}^{(0)} \equiv \bar{Y}^{(0)} \bmod \pi_{\alpha}^{1 /(q-1)} m_{\mathrm{sep}}
$$

for all solutions $\bar{Y}^{(0)}$ of the system (11), and this means that $\tau \in \operatorname{Gal}\left(K_{\mathrm{sep}} / L_{\alpha}\right)$.

The corollary is proved. 


\section{FALTings's STRICT MODULES}

5.2.4 For any finite extension $F \subset F_{1}$ in $K_{\text {sep }}$, let $v\left(F_{1} / F\right)$ be the minimal rational number such that the ramification groups $\Gamma_{F}^{(v)}$ act trivially on $F_{1}$ if $v>v\left(F_{1} / F\right)$.

Proposition. With the notation from $\S 5.2 .3$ there is the following inequality

$$
v(L / K) \leqslant e\left(N+\frac{1}{q-1}\right)-1
$$

Proof. Suppose that this inequality does not hold. Then there is a rational number $\alpha=m /\left(q^{M}-1\right)$ satisfying the assumptions from the beginning of n.5.2.2 and the inequalities

$$
v(L / K)>\alpha>\alpha\left(1-\frac{1}{q^{M}}\right)>e\left(N+\frac{1}{q-1}\right)-1 .
$$

(We can always choose a sufficiently large $M$.)

Then by the lemma of $\S 5.2 .3$ one has $L_{\alpha}=L K_{\alpha}$ and this implies that

$$
v\left(L_{\alpha} / K\right)=\max \left\{v(L / K), v\left(K_{\alpha} / K\right)\right\}=v(L / K) .
$$

On the other hand, looking at the maximal edge points of Herbrand functions we obtain from the composition property $\varphi_{L_{\alpha} / K}=\varphi_{L_{\alpha} / K_{\alpha}} \circ \varphi_{K_{\alpha} / K}$ that

$$
\begin{aligned}
v\left(L_{\alpha} / K\right) & =\max \left\{v\left(K_{\alpha} / K\right), \varphi_{K_{\alpha} / K}\left(v\left(L_{\alpha} / K_{\alpha}\right)\right)\right\} \\
& =\max \left\{\alpha, \frac{v\left(L_{\alpha} / K_{\alpha}\right)-\alpha}{q}+\alpha\right\}<v\left(L_{\alpha} / K_{\alpha}\right),
\end{aligned}
$$

because $\alpha<v(L / K)=v\left(L_{\alpha} / K_{\alpha}\right)$. However, this contradicts (13).

Theorem 8 is proved.

5.3 As was noticed in $\S 3.5 .1$, if $e=1$ then strict $O$-modules that are killed by $[\pi]$ behave very similarly to group schemes of period $p$ over Witt vectors. For this reason, one can apply directly methods from [Abr87b] to prove the following result.

Theorem 9. Suppose that $q \geqslant 4$ and $H$ is an $\mathbb{F}_{q}\left[\Gamma_{K}\right]$-module such that:

(a) the action of the inertia subgroup of $\Gamma_{K}$ on the semisimple envelope of $H$ is given by characters, which satisfy Serre's conjecture, cf. Theorem 7;

(b) the ramification subgroups $\Gamma_{K}^{(v)}$ act trivially on $H$ if $v>1 /(q-1)$ (i.e. the ramification estimate from Theorem 8 holds for $H$ ).

Then there is a $\mathcal{G}=\left(G, G^{b}\right) \in \operatorname{DGr}^{\prime}(O)_{R}$ such that $H \simeq G\left(K_{\text {sep }}\right)$.

\section{ACKNOWLEDGEMENT}

The author expresses his gratitude for hospitality to the Max-Planck-Institute in Mathematics where a part of this paper was written.

\section{REFERENCES}

Abr87a V. Abrashkin, Honda systems of group schemes of period p, Izv. Akad. Nauk SSSR Ser. Mat. 51 (1987), 451-484. (Engl. transl. Math. USSR Izv. 30 (1988), 419-453.)

Abr87b V. Abrashkin, Galois modules of period p group schemes over the ring of Witt vectors, Izv. Akad. Sci. SSSR Ser. Mat. 51 (1987), 691-736. (Engl. transl. Math. USSR Izv. 31 (1988), 1-46.)

Abr90 V. Abrashkin, Group schemes over a discrete valuation ring with small ramification, Algebra i Analiz 1 (1989), 60-95. (Engl. transl. Leningrad Math. J. 1 (1990), 57-97.) 


\section{FALTingS'S STRICT MODULES}

Abr98 V. Abrashkin, The ramification filtration of the Galois group of a local field. III, Izv. Ross. Akad. Nauk Ser. Mat. 62 (1998), 3-48. (Engl. transl. Izv. Math. 62 (1998), 857-900.)

Bre00 C. Breuil, Groupes p-divisibles, groupes finis et modules filtres, Ann. of Math. (2) 152 (2000), 489-549.

Con99 B. Conrad, Finite group schemes over bases with low ramification, Compositio Math. 119 (1999), 239-320.

DG70 M. Demazure and P. Gabriel, Groupes algébriques. Tome 1: géométrie algébrique, généralités, groupes commutatifs (North-Holland, Amsterdam, 1970).

Fal02 G. Faltings, Group schemes with strict $\mathcal{O}$-action, Moscow Math. J. 2 (2002), 249-279.

Fon75 J. M. Fontaine, Groupes finis commutatifs sur les vecteurs de Witt, C. R. Acad. Sci. Paris Sér. A-B 280 (1975), A1423-A1425.

Fon85 J.-M. Fontaine, Il n’y a pas de variété abélienne sur $\mathbb{Z}$, Invent. Math. 81 (1985), 515-538.

Fon90 J.-M. Fontaine, Représentations p-adiques des corps locaux. I, in The Grothendieck Festschrift, vol. II, Progress in Mathematics, vol. 87 (Birkhäuser, Boston, MA, 1990), 249-309.

Fon93 J.-M. Fontaine, Schémas propres et lisses sur $\mathbb{Z}$, in Proceedings of the Indo-French Conference on Geometry (Bombay, 1989) (Hindustan Book Agency, Delhi, 1993), 43-56.

Gab70 P. Gabriel, Étude infinitésimale des schémas en groupes, in Schémas en Groupes I, Lecture Notes in Mathematics, vol. 151 (Springer, Berlin, 1970), 474-560.

Gen96 A. Genestier, Espaces symmetriques de Drinfeld, Astérisque 234 (1996).

Gib04 W. Gibbons, Dieudonné theory for Faltings's strict modules, PhD thesis, University of Durham, September (2004).

Ray74 M. Raynaud, Schémas en groupes de type $(p, \ldots, p)$, Bull. Soc. Math. France 102 (1974), 241-280.

Tag92 Yu. Taguchi, Ramification arising from Drinfeld modules, in The Arithmetic of Function Fields. Proceedings of the Workshop (Ohio State University, 1991) (de Gruyter, Berlin, 1992), 171-188.

Tag95 Yu. Taguchi, A duality for finite t-modules, J. Math. Sci. Univ. Tokyo 2 (1995), 563-588.

Tat67 J. Tate, p-divisible groups, in Proc. conf. local fields (Driebergen, 1966) (Springer, Berlin, 1967), 158-183.

V. Abrashkin victor.abrashkin@durham.ac.uk

Steklov Mathematics Institute, Gubkina 8, 117966 Moscow, Russia

Current address: Department of Mathematics, University of Durham, Science Laboratories, Durham

DH1 3LE, UK 